Volume 8. No. 9, September 2020

International Journal of Emerging Trends in Engineering Research

Available Online at http://www.warse.org/IJETER/static/pdf/file/ijeter197892020.pdf

https://doi.org/10.30534/ijeter/2020/197892020

\title{
CNNIWPSO: Convolutional Neural Network Inertia Weight based Particle Swarm Optimization
}

\author{
Y.V.R. Naga Pawan ${ }^{1}$, Kolla Bhanu Prakash ${ }^{2}$ \\ ${ }^{1}$ Research Scholar, Department of CSE, Koneru Lakshmaiah Education Foundation, Green Fields, \\ Vaddeswaram, Guntur (Dist.), A.P., India, ynpawan@ gmail.com \\ ${ }^{2}$ Professor, Department of CSE, Koneru Lakshmaiah Education Foundation, Green Fields, Vaddeswaram, Guntur \\ (Dist.), A.P., India, drkbp@kluniversity.in
}

\begin{abstract}
Finding optimal solution is the prime moto of every optimization algorithm. Among various optimization algorithms Particle Swarm Optimization (PSO) earned a place as an optimization problem solving algorithm, due to its simplicity. Many researchers worked on PSO in improving the efficiency of the PSO. By tuning Inertia Weight, PSO converge to the optimal solution. In this paper a new inertia weight tuning PSO, Convolutional Neural Network based Inertia Weight Particle Swarm Optimization (CNNIWPSO) is proposed. The performance of the CNNIWPSO is compared with Constant Inertia Weight PSO (CIWPSO), Random Inertia Weight PSO (RWIPSO) and Linearly Decreasing Inertia Weight PSO (LDIWPSO). The results shows that CNNIWPSO is outperformed.
\end{abstract}

Key words : Particle Swarm Optimization, Inertia Weight, Convolutional Neural Network, Benchmark Functions, Convergence.

\section{INTRODUCTION}

The conventional computational techniques are unable to solve many complex problems. In the year 1995, Eberhart and Kennedy proposed an evolutionary computing technique, Particle Swarm Optimization (PSO) [1], for solving metaheuristic and stochastic problems. PSO is inspired by flock of birds, fish schooling. PSO is applied successfully in wide range of problems [3]-[8]. PSO can be applied to non-differentiable, non-linear, huge search space problems and gives the better results with a good efficiency [9].

In a PSO, the particle fly through the search space. Each particle is a candidate solution. The fitness function measures the performance of the particle. Different problems has different fitness functions. The birds find their food by randomly follow one of the members in the group which are in the nearest position to the food. The birds communicate each other to achieve best position. This process is iterated till food is found [10].

\section{BASIC PSO ALGORITHM}

In the Basic PSO (BPSO), a Swarm, $\mathrm{S}$, consists of n particles represented as $S=\left\{P_{1}, P_{2}, P_{3}, \ldots, P_{n}\right\}$. Each Particle $P_{i}$ has a position in the search space represented by $\mathrm{PX}_{\mathrm{i}}=\left\{\mathrm{px}_{\mathrm{il}}, \mathrm{px}_{\mathrm{i} 2}\right.$, $\left.\ldots, \mathrm{px}_{\mathrm{iD}}\right\}$, where $\mathrm{D}$ is $\mathrm{D}$-dimensional search space. In the search space, each particle $P_{i}$, moves with a velocity $V_{i}$, represented as $P V_{i}=\left\{\mathrm{pv}_{\mathrm{i} 1}, \mathrm{pv}_{\mathrm{i} 2}, \mathrm{pv}_{\mathrm{i} 3}, \ldots, \mathrm{pv}_{\mathrm{iD}}\right\}$. Each particle, $\mathrm{P}_{\mathrm{i}}$, maintains its best position, $P b_{i}$, represented as $P b_{i}=\left\{p b_{i 1}\right.$, $\left.\mathrm{pb}_{\mathrm{i} 2}, \ldots, \mathrm{pb}_{\mathrm{iD}}\right\}$. Amon $\mathrm{g}$ the population of all particles, the best particle is determined and represented as $P_{g}=\left\{\mathrm{pg}_{1}, \mathrm{pg}_{2}, \mathrm{pg}_{3}\right.$, $\left.\ldots, \mathrm{pg}_{\mathrm{D}}\right\}$. The basic equations with functioning of BPSO are given by (1) and (2).

$p v_{i d}=p v_{i d}+c_{1} * \operatorname{random}() *\left(p b_{i}-p x_{i d}\right)+c_{2} * \operatorname{Random}() *$

$\left(p g_{i}-p x_{\mathrm{id}}\right)$

$p x_{i d}=p x_{i d}+p v_{i d}$

where $\mathrm{c} 1$ and $\mathrm{c} 2$ are two acceleration coefficients, random() and $\operatorname{Random}()$ are two random functions in the $[0,1] \cdot \mathrm{pv}_{\mathrm{i}}$ is the clamped to a maximum velocity $\mathrm{pv}_{\max }$, the parameter given by the user. The first part of the (1) represents previous velocity, the second part is the cognition part of the particle, and the third part represents the cooperation among the particles [11].

As particles tends to explore the search space hugely, the velocities of the particles are limited to the constant $\mathrm{pv}_{\max }$ [12]. The particle velocity is adjusted using

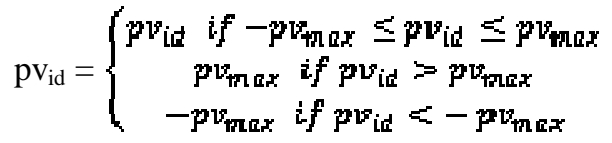

The value for $\mathrm{pv}_{\max }$ is typically chosen as a fraction of the search space dimension shown as (4) [12] - [13], where $\delta$ is the velocity clamping factor.

$\mathrm{pv}_{\max }=\delta\left(\mathrm{px}_{\max }-\mathrm{px}_{\min }\right)$ where $\delta \in(0,1)$.

As the search space is bounded by the interval $\left[\mathrm{px}_{\min }, \mathrm{px}_{\max }\right]$, the velocity clamping [14] of the particle is in the interval $\left[-\mathrm{pv}_{\max }, \mathrm{pv}_{\max }\right]$, where $\mathrm{pv}_{\max }=\delta *\left(\mathrm{px}_{\max }-\mathrm{px}_{\min }\right) / 2$. 
Y.V.R. Naga Pawan et al., International Journal of Emerging Trends in Engineering Research, 8(9), September 2020, 6111 - 6130

The flowchart for the BPSO is shown below i.e., in Figure 1:

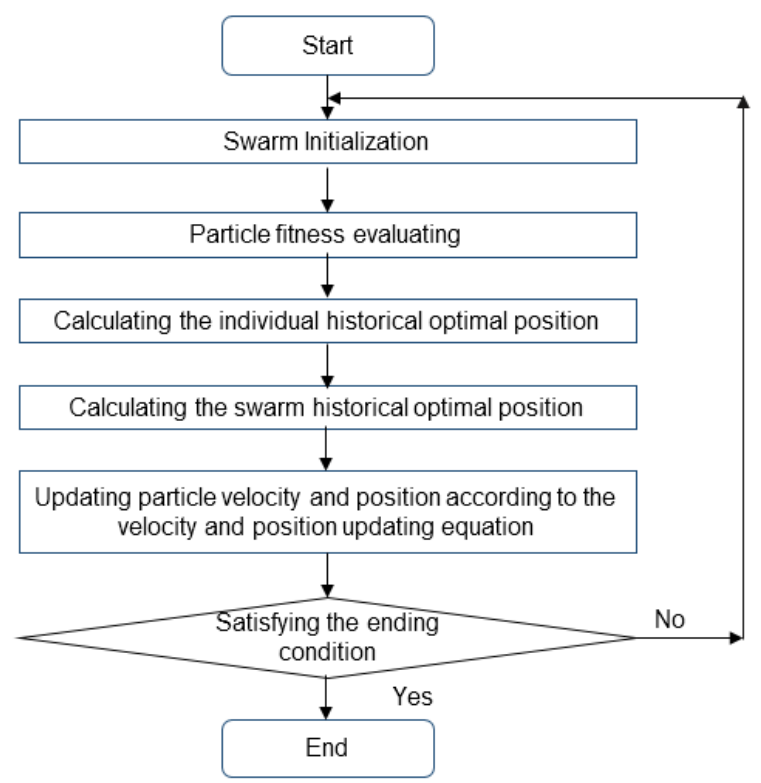

Figure 1: Flowchart of Basic PSO

The flowchart has three parts, the first part is local, second part is based on vicinity and the last part on global.

The pseudocode for Basic PSO is given below:

Step 1:

Initialization

For each particle, $\mathrm{P}_{\mathrm{i}}$, in the population

Initialize $\mathrm{px}_{\mathrm{i}}$ with uniform distribution

Initialize $\mathrm{pv}_{\mathrm{i}}$ randomly.

Evaluate the objective function of $\mathrm{px}_{\mathrm{i}}$ and assigned the value to fitness[i].

Initialize pbest $\mathrm{i}_{\mathrm{i}}$ with a copy of $\mathrm{px}_{\mathrm{i}}$.

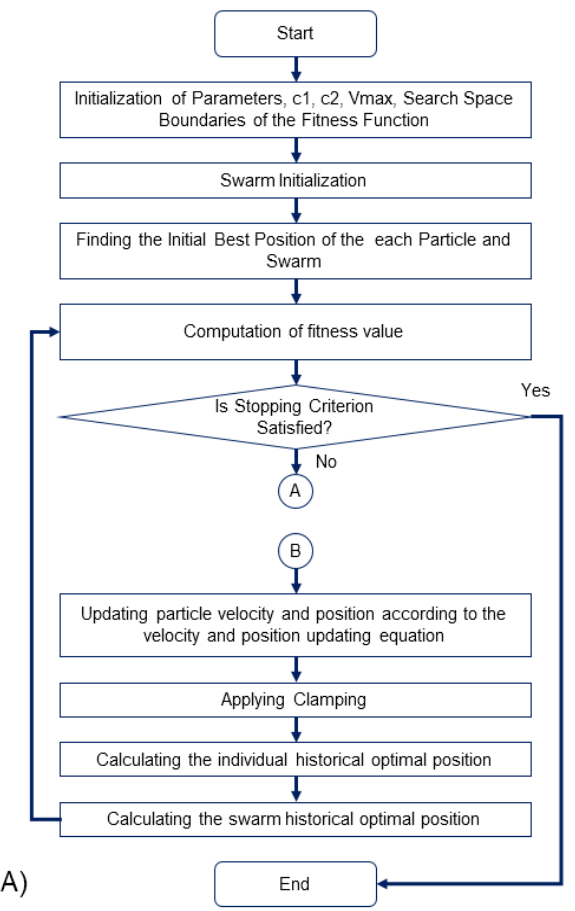

Initialize pbest_ftness $s_{i}$ with a copy of fitness ${ }_{i}$.

Initialize pgbest with index of the particle with the least fitness.

Step 2:

Repeat until stopping criterion is reached

For each particle, $\mathrm{P}_{\mathrm{i}}$,

Update $\mathrm{pv}_{\mathrm{i}}$ and $\mathrm{px}_{\mathrm{i}}$ according to the equations (1) and (2)

Evaluate fitness ${ }_{i}$

If fitness $\mathrm{i}_{\mathrm{i}}<$ pbest_fitness $_{\mathrm{i}}$ then

Pbest $_{\mathrm{i}}=\mathrm{px}_{\mathrm{i}}$

Pbest_fitness ${ }_{\mathrm{i}}=$ fitness $_{\mathrm{i}}$

Update pgbest by the particle with current least fitness among the population

Figure 2: Pseudocode of Basic PSO

\section{INERTIA WEIGHT BASED PSO}

Shi and Eberhart [2], developed Inertia Weight Based PSO to control exploration and exploitation. Equation (1) resulting as (5).

$p v_{i d}=w * p v_{i d}+c_{1} * \operatorname{random}() *\left(p b_{i}-p x_{i d}\right)+c_{2} *$

$\operatorname{Random}() *\left(p g_{i}-p x_{\mathrm{id}}\right)$

(5)

J.C.Bansal et al [15] discussed various strategies of Inertia Weight Based PSO. Constant Inertia Weight PSO (CIWPSO) [15], Random Inertia Weight PSO (RIWPSO) [17] and Linear Decreasing Inertia Weight PSO (LDIWPSO) [18] are considered for comparison with proposed Convolutional Neural Network Inertia Weight PSO (CNNIWPSO). In [19]-[21], it is discussed about setting of parameters for a PSO for reaching optimization solution.

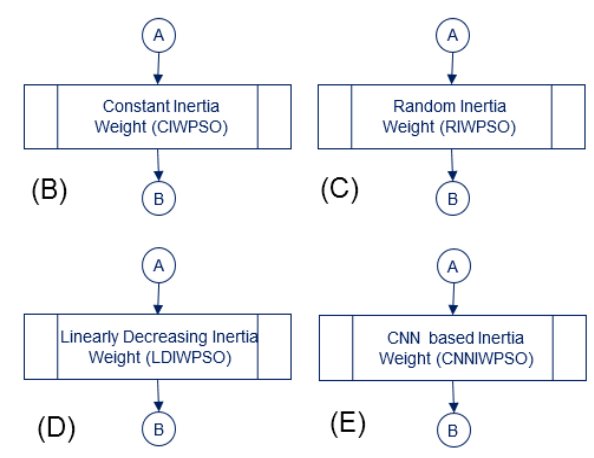

Figure 3: (A) and (B) shows the implementation CIWPSO, (A) and (C) shows the implementation of RIWPSO, (A) and (D) shows the implementation of LDIWPSO and, (A) and (E) depicts the CNNIWPSO. 


\section{Convolutional Neural Network Inertia Weight PSO (CNNIWPSO)}

In CNNIWPSO, the new inertia weight is computed using univariate Convolutional Neural Network. Initially, CNN is trained with different inertia weights from 0.05 to 1.00 . In every iteration new inertia weight is predicted. The predicted IW is used to move the swarm using (5) and (2). The process is terminated when stopping criterion is reached.

\section{EXPERIMENTAL RESULTS}

Experiments are conducted with different Inertia Weight based PSOs, CIWPSO, RIWPSO, LDIWPSO and CNNIWPSO over different optimization test problems.

\section{A. Parameter Setting}

Swarm sizes with 50, 75 and 100 particles with different dimensions, 10, 15 and 25 are considered for conducting experiments. In total, 15 simulations are run to reduce the effect of the randomness.

\section{B. Results}

The results are collected from the perspective of best error, mean error, variance, standard deviation, mean square error, root mean square error, mean iterations and mean time taken (in seconds) for the simulations. The results are tabulated from table 2 to table 9 and plotted from the fig 4 to fig 11 . The details about the benchmark functions are shown in Table 1 which are used for computing fitness.

Table 1: Benchmark Functions

\begin{tabular}{|c|c|c|c|c|}
\hline $\begin{array}{l}\text { Benchmar } \\
\text { k Function } \\
\text { name }\end{array}$ & Properties & Benchmark Function & Interval & $\begin{array}{c}\text { Best } \\
\text { fitness } \\
\text { value at }\end{array}$ \\
\hline Ackley & $\begin{array}{l}\text { n-dimensional, } \\
\text { continuous, } \\
\text { multimodal, } \\
\text { non-convex, } \\
\text { differentiable }\end{array}$ & 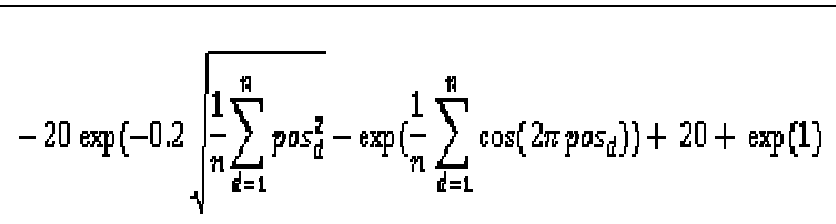 & {$[-32,+32]$} & $f(0)=0$ \\
\hline Alpine & $\begin{array}{l}\text { n-dimensional, } \\
\text { non-separable, } \\
\text { multimodal, } \\
\text { non-convex, } \\
\text { differentiable }\end{array}$ & $\sum_{d=1}^{\pi}\left|p a s_{d}, \sin \left(p o s_{d}\right)+0.1 \operatorname{pos}_{d}\right|$ & {$[0,10]$} & $f(0)=0$ \\
\hline Rastrigin & $\begin{array}{l}\text { n-dimensional, } \\
\text { continuous, } \\
\text { differentiable, } \\
\text { separable, } \\
\text { multimodal, convex }\end{array}$ & $10 . n+\sum_{d=1}^{n}\left[p o s_{d}^{2}-10 \cdot \cos \left(2 \pi p o s_{d}\right)\right]$ & {$[-5.12,+5.12[$} & $f(0)=0$ \\
\hline Rosenbrock & $\begin{array}{l}\text { continuous, } \\
\text { differentiable, } \\
\text { non-separable, } \\
\text { multimodal, } \\
\text { non-convex }\end{array}$ & $\sum_{d=1}^{n}\left[100 \cdot\left(p 0 s_{d+1}-p 0 s_{d}^{2}\right)^{2}+\left(1-p 0 s_{d}\right)^{2}\right]$ & {$[-5,10]$} & $f(1)=0$ \\
\hline Sphere & $\begin{array}{l}\text { n-dimensional, } \\
\text { continuous, convex, } \\
\text { differentiable, } \\
\text { unimodal, separable }\end{array}$ & $\sum_{d=1}^{n} \operatorname{pos}_{d}^{2}$ & {$[-5.12,+5.12]$} & $f(0)=0$ \\
\hline
\end{tabular}


Y.V.R. Naga Pawan et al., International Journal of Emerging Trends in Engineering Research, 8(9), September 2020, 6111 - 6130

Table 2: Computed Best Error for PSOs with respect different Swarm Sizes and Dimensions. (Figure 4)

\begin{tabular}{|c|c|c|c|c|c|c|}
\hline \multirow{2}{*}{ Swarm Size } & \multirow{2}{*}{ Dimension } & \multirow{2}{*}{ BMF } & \multicolumn{4}{|c|}{ PSOs } \\
\hline & & & CIWPSO & RIWPSO & LDIWPSO & CNNIWPSO \\
\hline \multirow{15}{*}{50} & \multirow{5}{*}{10} & Ackley & $1.0000 \mathrm{E}-05$ & $1.0000 \mathrm{E}-05$ & $1.0000 \mathrm{E}-05$ & $3.2000 \mathrm{E}-05$ \\
\hline & & Alpine & $0.0000 \mathrm{E}+00$ & $0.0000 \mathrm{E}+00$ & $0.0000 \mathrm{E}+00$ & $0.0000 \mathrm{E}+00$ \\
\hline & & Rastrigin & $8.0000 \mathrm{E}-06$ & $1.0000 \mathrm{E}-05$ & $9.0000 \mathrm{E}-06$ & 8.0000E-06 \\
\hline & & Rosenbrock & 6.0000E-06 & 8.0000E-06 & $7.0000 \mathrm{E}-06$ & $6.0000 \mathrm{E}-06$ \\
\hline & & Sphere & $9.0000 \mathrm{E}-06$ & $1.0000 \mathrm{E}-05$ & $8.0000 \mathrm{E}-06$ & $1.0000 \mathrm{E}-05$ \\
\hline & \multirow{5}{*}{15} & Ackley & $1.5640 \mathrm{E}-03$ & $1.4158 \mathrm{E}-02$ & $8.0190 \mathrm{E}-03$ & $1.3987 \mathrm{E}-02$ \\
\hline & & Alpine & $0.0000 \mathrm{E}+00$ & $0.0000 \mathrm{E}+00$ & $0.0000 \mathrm{E}+00$ & $0.0000 \mathrm{E}+00$ \\
\hline & & Rastrigin & $3.7000 \mathrm{E}-05$ & 4.8000E-05 & $2.7500 \mathrm{E}-04$ & $2.9600 \mathrm{E}-04$ \\
\hline & & Rosenbrock & $1.3000 \mathrm{E}-05$ & $1.8000 \mathrm{E}-05$ & $5.8000 \mathrm{E}-05$ & $1.2500 \mathrm{E}-04$ \\
\hline & & Sphere & $7.8000 \mathrm{E}-05$ & $6.4000 \mathrm{E}-05$ & $9.3000 \mathrm{E}-05$ & $8.5600 \mathrm{E}-04$ \\
\hline & \multirow{5}{*}{25} & Ackley & $1.7884 \mathrm{E}-01$ & $4.1187 \mathrm{E}-01$ & 4.1186E-01 & $1.1538 \mathrm{E}+00$ \\
\hline & & Alpine & $0.0000 \mathrm{E}+00$ & $0.0000 \mathrm{E}+00$ & $0.0000 \mathrm{E}+00$ & $0.0000 \mathrm{E}+00$ \\
\hline & & Rastrigin & $1.8620 \mathrm{E}-03$ & 4.2530E-03 & $1.8550 \mathrm{E}-02$ & $4.6820 \mathrm{E}-03$ \\
\hline & & Rosenbrock & $2.7300 \mathrm{E}-04$ & $6.1300 \mathrm{E}-04$ & $1.6680 \mathrm{E}-03$ & $2.8840 \mathrm{E}-03$ \\
\hline & & Sphere & $1.4920 \mathrm{E}-03$ & $3.5220 \mathrm{E}-03$ & $1.0627 \mathrm{E}-02$ & $1.1868 \mathrm{E}-02$ \\
\hline \multirow{15}{*}{75} & \multirow{5}{*}{10} & Ackley & $9.0000 \mathrm{E}-06$ & $9.0000 \mathrm{E}-06$ & $9.0000 \mathrm{E}-06$ & $9.0000 \mathrm{E}-06$ \\
\hline & & Alpine & $0.0000 \mathrm{E}+00$ & $0.0000 \mathrm{E}+00$ & $0.0000 \mathrm{E}+00$ & $0.0000 \mathrm{E}+00$ \\
\hline & & Rastrigin & $7.0000 \mathrm{E}-06$ & $7.0000 \mathrm{E}-06$ & $7.0000 \mathrm{E}-06$ & $8.0000 \mathrm{E}-06$ \\
\hline & & Rosenbrock & 7.0000E-06 & $7.0000 \mathrm{E}-06$ & $5.0000 \mathrm{E}-06$ & $8.0000 \mathrm{E}-06$ \\
\hline & & Sphere & 8.0000E-06 & 8.0000E-06 & $9.0000 \mathrm{E}-06$ & $8.0000 \mathrm{E}-06$ \\
\hline & \multirow{5}{*}{15} & Ackley & 8.4400E-04 & $2.4900 \mathrm{E}-04$ & $2.2180 \mathrm{E}-03$ & $1.5890 \mathrm{E}-03$ \\
\hline & & Alpine & $0.0000 \mathrm{E}+00$ & $0.0000 \mathrm{E}+00$ & $0.0000 \mathrm{E}+00$ & $0.0000 \mathrm{E}+00$ \\
\hline & & Rastrigin & $1.0000 \mathrm{E}-05$ & $1.2000 \mathrm{E}-05$ & $1.0000 \mathrm{E}-05$ & $3.2000 \mathrm{E}-05$ \\
\hline & & Rosenbrock & $9.0000 \mathrm{E}-06$ & $2.0000 \mathrm{E}-05$ & $1.0000 \mathrm{E}-05$ & $7.0000 \mathrm{E}-06$ \\
\hline & & Sphere & $1.0000 \mathrm{E}-05$ & $2.5000 \mathrm{E}-05$ & $5.2000 \mathrm{E}-05$ & $6.7000 \mathrm{E}-05$ \\
\hline & \multirow{5}{*}{25} & Ackley & 2.8523E-02 & $5.7368 \mathrm{E}-02$ & $5.9241 E-02$ & $2.1002 \mathrm{E}-01$ \\
\hline & & Alpine & $0.0000 \mathrm{E}+00$ & $0.0000 \mathrm{E}+00$ & $0.0000 \mathrm{E}+00$ & $0.0000 \mathrm{E}+00$ \\
\hline & & Rastrigin & $1.0300 \mathrm{E}-03$ & $1.6660 \mathrm{E}-03$ & $3.0060 \mathrm{E}-03$ & $1.3860 \mathrm{E}-02$ \\
\hline & & Rosenbrock & 8.0000E-05 & $2.4300 \mathrm{E}-04$ & $9.3600 \mathrm{E}-04$ & $1.9030 \mathrm{E}-03$ \\
\hline & & Sphere & $4.9100 \mathrm{E}-04$ & $1.4320 \mathrm{E}-03$ & $5.8980 \mathrm{E}-03$ & $1.4345 \mathrm{E}-02$ \\
\hline \multirow{15}{*}{100} & \multirow{5}{*}{10} & Ackley & $9.0000 \mathrm{E}-06$ & $9.0000 \mathrm{E}-06$ & $5.0000 \mathrm{E}-06$ & $8.0000 \mathrm{E}-06$ \\
\hline & & Alpine & $0.0000 \mathrm{E}+00$ & $0.0000 \mathrm{E}+00$ & $0.0000 \mathrm{E}+00$ & $0.0000 \mathrm{E}+00$ \\
\hline & & Rastrigin & $9.0000 \mathrm{E}-06$ & $7.0000 \mathrm{E}-06$ & $7.0000 \mathrm{E}-06$ & $7.0000 \mathrm{E}-06$ \\
\hline & & Rosenbrock & 8.0000E-06 & $8.0000 \mathrm{E}-06$ & $7.0000 \mathrm{E}-06$ & $5.0000 \mathrm{E}-06$ \\
\hline & & Sphere & 8.0000E-06 & 4.0000E-06 & 8.0000E-06 & $8.0000 \mathrm{E}-06$ \\
\hline & \multirow{5}{*}{15} & Ackley & $1.0000 \mathrm{E}-05$ & $5.4400 \mathrm{E}-04$ & $1.1900 \mathrm{E}-04$ & $1.1800 \mathrm{E}-03$ \\
\hline & & Alpine & $0.0000 \mathrm{E}+00$ & $0.0000 \mathrm{E}+00$ & $0.0000 \mathrm{E}+00$ & $0.0000 \mathrm{E}+00$ \\
\hline & & Rastrigin & $1.0000 \mathrm{E}-05$ & $1.6000 \mathrm{E}-05$ & $9.0000 \mathrm{E}-06$ & $1.0000 \mathrm{E}-05$ \\
\hline & & Rosenbrock & $1.0000 \mathrm{E}-05$ & $9.0000 \mathrm{E}-06$ & $9.0000 \mathrm{E}-06$ & $1.0000 \mathrm{E}-05$ \\
\hline & & Sphere & $1.0000 \mathrm{E}-05$ & $1.0000 \mathrm{E}-05$ & $9.0000 \mathrm{E}-06$ & $1.8000 \mathrm{E}-05$ \\
\hline & \multirow{5}{*}{25} & Ackley & $1.3386 \mathrm{E}-02$ & 4.7314E-02 & $5.9322 \mathrm{E}-02$ & $6.5859 \mathrm{E}-02$ \\
\hline & & Alpine & $0.0000 \mathrm{E}+00$ & $0.0000 \mathrm{E}+00$ & $0.0000 \mathrm{E}+00$ & $0.0000 \mathrm{E}+00$ \\
\hline & & Rastrigin & $5.2700 \mathrm{E}-04$ & $1.3260 \mathrm{E}-03$ & $1.8870 \mathrm{E}-03$ & $2.2400 \mathrm{E}-03$ \\
\hline & & Rosenbrock & 4.9000E-05 & $5.8000 \mathrm{E}-05$ & $5.0200 \mathrm{E}-04$ & $9.5500 \mathrm{E}-04$ \\
\hline & & Sphere & $4.7000 \mathrm{E}-04$ & $2.8300 \mathrm{E}-04$ & $2.5600 \mathrm{E}-03$ & $8.7190 \mathrm{E}-03$ \\
\hline
\end{tabular}


Comparison of PSOs with Best Error
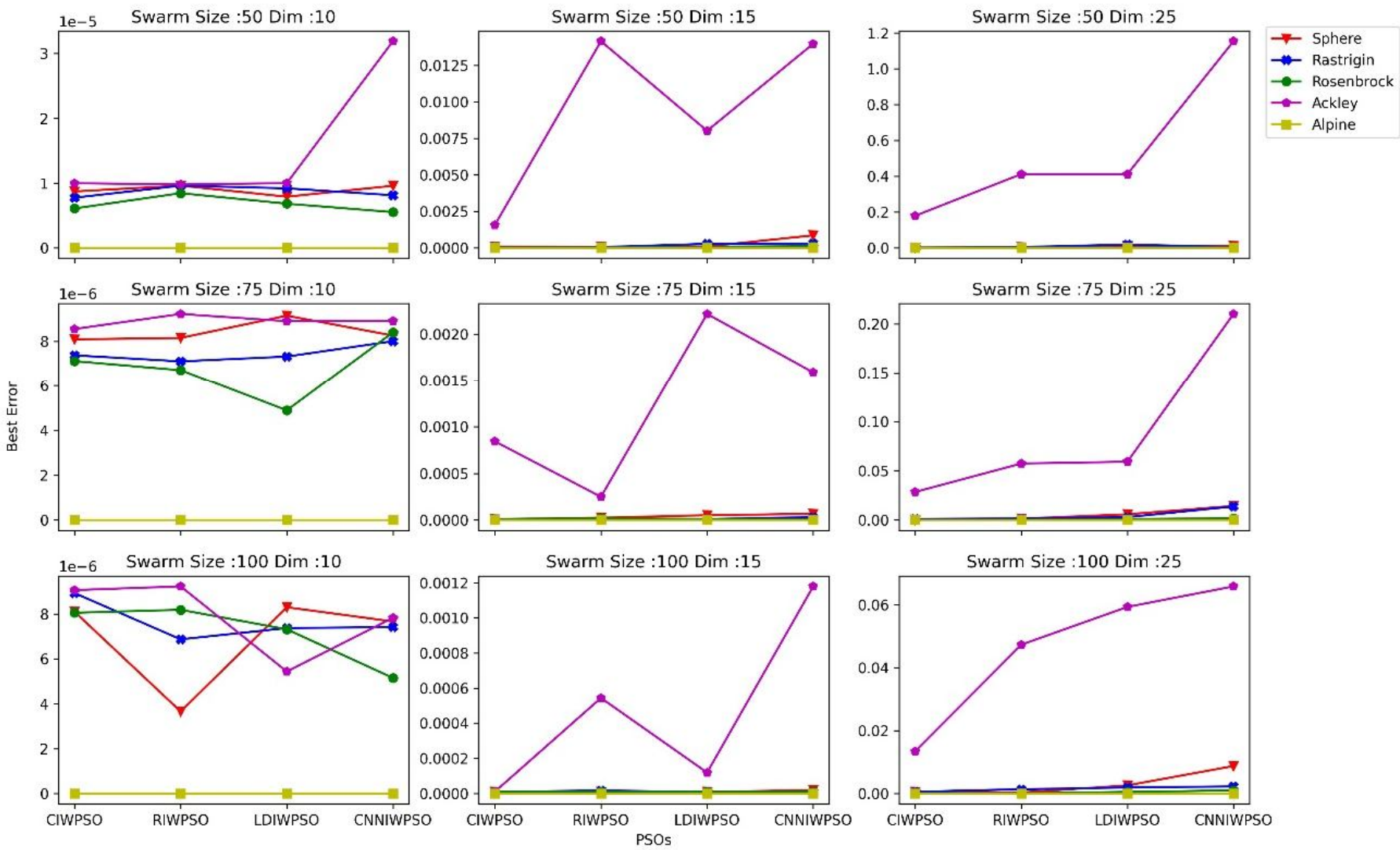

Figure 4: Best Error computed for the swarm size of 50, 75 and 100 with dimensions 10, 15 and 25

From Table 2 and Figure 4, for the benchmark functions, Ackley, Rosenbrock and sphere, it is observed that the best error obtained for CNNWPSO is comparatively nearer to the other models convergence. For Alpine, the CNNIWPSO is converged as other models. For Rastrigin, with increasing swarm sizes and dimensions, the best error obtained is notable when compared with other models. 
Y.V.R. Naga Pawan et al., International Journal of Emerging Trends in Engineering Research, 8(9), September 2020, 6111 - 6130

Table 3: Computed Mean Error for PSOs with respect different Swarm Sizes and Dimensions. (Figure 5)

\begin{tabular}{|c|c|c|c|c|c|c|}
\hline \multirow{2}{*}{ Swarm Size } & \multirow{2}{*}{ Dimension } & \multirow{2}{*}{ BMF } & \multicolumn{4}{|c|}{ PSOs } \\
\hline & & & CIWPSO & RIWPSO & LDIWPSO & CNNIWPSO \\
\hline \multirow{15}{*}{50} & \multirow{5}{*}{10} & Ackley & $3.6492 \mathrm{E}+00$ & $4.8287 \mathrm{E}+00$ & $7.7269 \mathrm{E}+00$ & $2.3888 \mathrm{E}+00$ \\
\hline & & Alpine & $5.2831 \mathrm{E}+01$ & $4.6940 \mathrm{E}+01$ & $5.4715 \mathrm{E}+01$ & $4.2141 \mathrm{E}+01$ \\
\hline & & Rastrigin & 1.9491E-01 & $1.4830 \mathrm{E}-01$ & $2.5884 \mathrm{E}-01$ & 9.9415E-02 \\
\hline & & Rosenbrock & $5.4788 \mathrm{E}-02$ & $3.2068 \mathrm{E}-02$ & $6.8679 \mathrm{E}-02$ & $2.6618 \mathrm{E}-02$ \\
\hline & & Sphere & 1.6950E-01 & $1.2870 \mathrm{E}-01$ & $2.8420 \mathrm{E}-01$ & $9.3068 \mathrm{E}-02$ \\
\hline & \multirow{5}{*}{15} & Ackley & $9.0266 \mathrm{E}+00$ & $1.2882 \mathrm{E}+01$ & $1.7771 \mathrm{E}+01$ & $7.5579 \mathrm{E}+00$ \\
\hline & & Alpine & $9.5725 \mathrm{E}+01$ & $8.4866 \mathrm{E}+01$ & $1.0453 \mathrm{E}+02$ & $6.8968 \mathrm{E}+01$ \\
\hline & & Rastrigin & 2.2103E-01 & 3.9361E-01 & $4.6333 \mathrm{E}-01$ & $1.9228 \mathrm{E}-01$ \\
\hline & & Rosenbrock & 4.4647E-02 & $5.3836 \mathrm{E}-02$ & 7.7879E-02 & $2.6976 \mathrm{E}-02$ \\
\hline & & Sphere & 2.8604E-01 & $2.8935 \mathrm{E}-01$ & $4.5313 \mathrm{E}-01$ & $1.7540 \mathrm{E}-01$ \\
\hline & \multirow{5}{*}{25} & Ackley & $3.2971 \mathrm{E}+01$ & $5.2092 \mathrm{E}+01$ & $5.4741 \mathrm{E}+01$ & $2.6451 \mathrm{E}+01$ \\
\hline & & Alpine & $1.3184 \mathrm{E}+02$ & $1.5629 \mathrm{E}+02$ & $1.4785 \mathrm{E}+02$ & $1.0170 \mathrm{E}+02$ \\
\hline & & Rastrigin & 7.9071E-01 & $1.1214 \mathrm{E}+00$ & $1.4719 \mathrm{E}+00$ & $6.2260 \mathrm{E}-01$ \\
\hline & & Rosenbrock & $1.2420 \mathrm{E}-01$ & $1.7996 \mathrm{E}-01$ & $2.2061 \mathrm{E}-01$ & $1.0929 \mathrm{E}-01$ \\
\hline & & Sphere & 7.7299E-01 & $1.1383 \mathrm{E}+00$ & $1.3934 \mathrm{E}+00$ & $6.5461 \mathrm{E}-01$ \\
\hline \multirow{15}{*}{75} & \multirow{5}{*}{10} & Ackley & $5.7835 \mathrm{E}+00$ & $3.8704 \mathrm{E}+00$ & $7.1575 \mathrm{E}+00$ & $3.6412 \mathrm{E}+00$ \\
\hline & & Alpine & $4.1195 \mathrm{E}+01$ & $5.0880 \mathrm{E}+01$ & $5.5471 \mathrm{E}+01$ & $4.4675 \mathrm{E}+01$ \\
\hline & & Rastrigin & $2.4224 \mathrm{E}-01$ & $2.0164 \mathrm{E}-01$ & $3.3316 \mathrm{E}-01$ & 3.2029E-01 \\
\hline & & Rosenbrock & 5.5133E-02 & $2.7863 \mathrm{E}-02$ & $5.6270 \mathrm{E}-02$ & $7.2341 \mathrm{E}-02$ \\
\hline & & Sphere & $1.9867 \mathrm{E}-01$ & $1.7552 \mathrm{E}-01$ & $3.6113 \mathrm{E}-01$ & $2.4171 \mathrm{E}-01$ \\
\hline & \multirow{5}{*}{15} & Ackley & $6.8274 \mathrm{E}+00$ & $1.0026 \mathrm{E}+01$ & $1.4303 \mathrm{E}+01$ & $4.9775 \mathrm{E}+00$ \\
\hline & & Alpine & $8.6647 \mathrm{E}+01$ & $1.0121 \mathrm{E}+02$ & $1.0323 \mathrm{E}+02$ & $6.6537 \mathrm{E}+01$ \\
\hline & & Rastrigin & $2.3147 \mathrm{E}-01$ & $2.7971 \mathrm{E}-01$ & $3.6850 \mathrm{E}-01$ & $1.2795 \mathrm{E}-01$ \\
\hline & & Rosenbrock & 4.1654E-02 & $3.8286 \mathrm{E}-02$ & $6.5292 \mathrm{E}-02$ & $2.1587 \mathrm{E}-02$ \\
\hline & & Sphere & 1.9299E-01 & $2.5492 \mathrm{E}-01$ & $3.8445 \mathrm{E}-01$ & $1.3245 \mathrm{E}-01$ \\
\hline & \multirow{5}{*}{25} & Ackley & $2.3358 \mathrm{E}+01$ & $3.5383 \mathrm{E}+01$ & $3.9869 \mathrm{E}+01$ & $1.8317 \mathrm{E}+01$ \\
\hline & & Alpine & $1.0377 \mathrm{E}+02$ & $1.4760 \mathrm{E}+02$ & $1.7023 \mathrm{E}+02$ & $1.1285 \mathrm{E}+02$ \\
\hline & & Rastrigin & $6.4827 \mathrm{E}-01$ & $8.5586 \mathrm{E}-01$ & $1.1381 \mathrm{E}+00$ & 4.4350E-01 \\
\hline & & Rosenbrock & 8.9687E-02 & $1.5305 \mathrm{E}-01$ & $1.7020 \mathrm{E}-01$ & 7.5689E-02 \\
\hline & & Sphere & $5.9860 \mathrm{E}-01$ & $9.1849 \mathrm{E}-01$ & $1.0890 \mathrm{E}+00$ & 4.6111E-01 \\
\hline \multirow{15}{*}{100} & \multirow{5}{*}{10} & Ackley & $4.7614 \mathrm{E}+00$ & $5.1894 \mathrm{E}+00$ & $8.6341 \mathrm{E}+00$ & $7.8956 \mathrm{E}+00$ \\
\hline & & Alpine & $6.1785 \mathrm{E}+01$ & $5.4256 \mathrm{E}+01$ & $6.1046 \mathrm{E}+01$ & $3.5174 \mathrm{E}+01$ \\
\hline & & Rastrigin & $1.7561 \mathrm{E}-01$ & $1.5128 \mathrm{E}-01$ & $2.9691 \mathrm{E}-01$ & $2.7655 \mathrm{E}-01$ \\
\hline & & Rosenbrock & 4.7669E-02 & $2.5870 \mathrm{E}-02$ & $5.5112 \mathrm{E}-02$ & $6.2122 \mathrm{E}-02$ \\
\hline & & Sphere & $2.3748 \mathrm{E}-01$ & $1.3589 \mathrm{E}-01$ & $3.3865 \mathrm{E}-01$ & 3.8471E-01 \\
\hline & \multirow{5}{*}{15} & Ackley & $6.3176 \mathrm{E}+00$ & $7.6248 \mathrm{E}+00$ & $1.2894 \mathrm{E}+01$ & $4.2287 \mathrm{E}+00$ \\
\hline & & Alpine & $1.0084 \mathrm{E}+02$ & $9.2082 \mathrm{E}+01$ & $9.8943 \mathrm{E}+01$ & $6.6237 \mathrm{E}+01$ \\
\hline & & Rastrigin & 1.8472E-01 & $1.9431 \mathrm{E}-01$ & $3.3833 \mathrm{E}-01$ & $1.1053 \mathrm{E}-01$ \\
\hline & & Rosenbrock & 3.7692E-02 & 4.4817E-02 & $6.9880 \mathrm{E}-02$ & $1.8866 \mathrm{E}-02$ \\
\hline & & Sphere & 2.1054E-01 & $2.3791 \mathrm{E}-01$ & $3.1015 \mathrm{E}-01$ & 1.1799E-01 \\
\hline & \multirow{5}{*}{25} & Ackley & $2.0562 \mathrm{E}+01$ & $2.5489 \mathrm{E}+01$ & 3.6697E+01 & $1.4501 \mathrm{E}+01$ \\
\hline & & Alpine & $1.3355 \mathrm{E}+02$ & $1.8061 \mathrm{E}+02$ & $1.6636 \mathrm{E}+02$ & $1.0216 \mathrm{E}+02$ \\
\hline & & Rastrigin & 4.7189E-01 & $6.4692 \mathrm{E}-01$ & $9.7412 \mathrm{E}-01$ & 3.9204E-01 \\
\hline & & Rosenbrock & 8.7962E-02 & $1.1687 \mathrm{E}-01$ & $1.3659 \mathrm{E}-01$ & $6.2085 \mathrm{E}-02$ \\
\hline & & Sphere & 5.0012E-01 & $5.6230 \mathrm{E}-01$ & $8.8067 \mathrm{E}-01$ & 3.7943E-01 \\
\hline
\end{tabular}


Y.V.R. Naga Pawan et al., International Journal of Emerging Trends in Engineering Research, 8(9), September 2020, 6111 - 6130

Comparison of PSOs with Mean Error
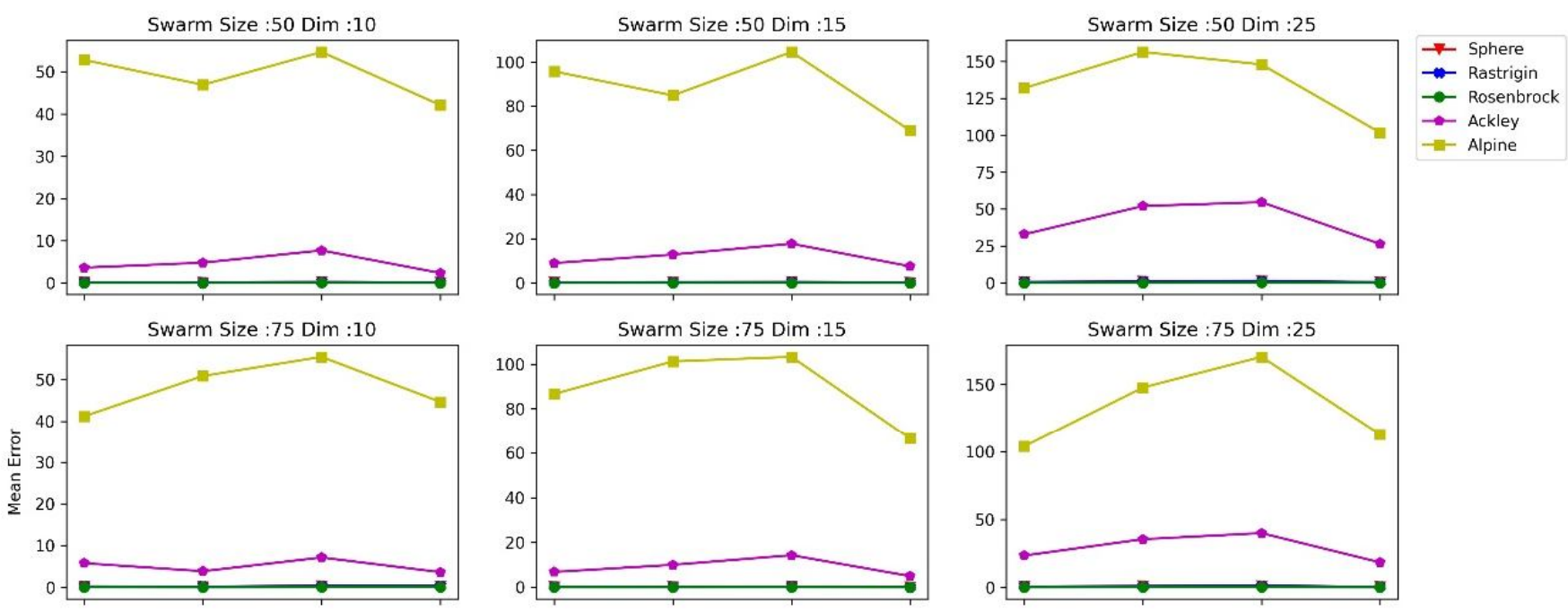

Swarm Size :75 Dim :25

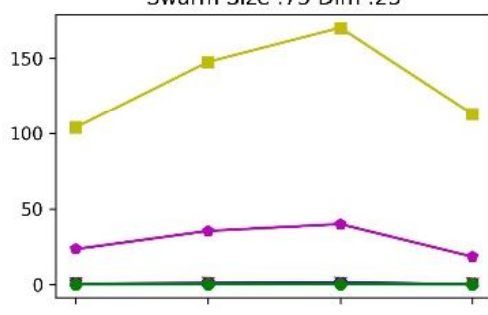

Swarm Size :100 Dim :10
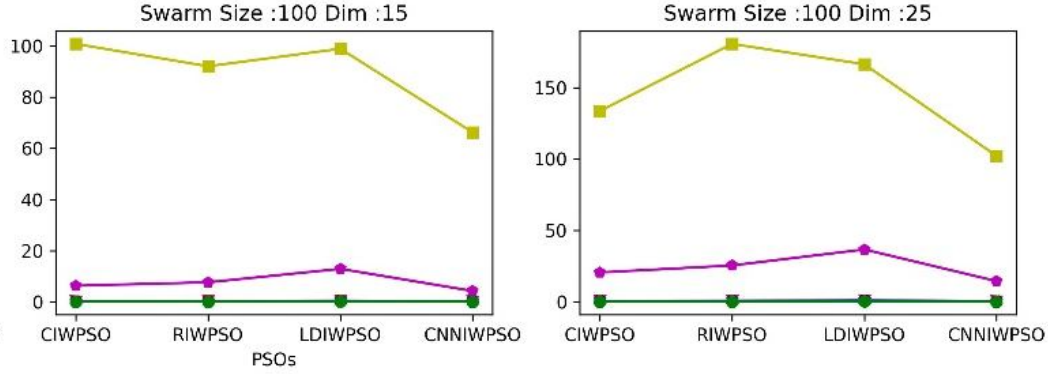

Figure 5: Mean Error computed for the swarm size of 50, 75 and 100 with dimensions 10, 15 and 25

From Table 3 and Figure 5, CNNIWPSO is outrival for the studied benchmark functions with increasing swarm sizes and dimensions, with respect to mean error 
Y.V.R. Naga Pawan et al., International Journal of Emerging Trends in Engineering Research, 8(9), September 2020, 6111 - 6130

Table 4: Computed Variance for PSOs with respect different Swarm Sizes and Dimensions. (Figure 6)

\begin{tabular}{|c|c|c|c|c|c|c|}
\hline \multirow{2}{*}{ Swarm Size } & \multirow{2}{*}{ Dimension } & \multirow{2}{*}{ BMF } & \multicolumn{4}{|c|}{ PSOs } \\
\hline & & & CIWPSO & RIWPSO & LDIWPSO & CNNIWPSO \\
\hline \multirow{15}{*}{50} & \multirow{5}{*}{10} & Ackley & $2.0742 \mathrm{E}+03$ & $2.3211 \mathrm{E}+03$ & $5.2282 \mathrm{E}+03$ & $8.5928 \mathrm{E}+02$ \\
\hline & & Alpine & $3.4779 \mathrm{E}+03$ & $2.7706 \mathrm{E}+03$ & $3.7017 \mathrm{E}+03$ & $2.7489 \mathrm{E}+03$ \\
\hline & & Rastrigin & $2.5551 \mathrm{E}+00$ & $2.4912 \mathrm{E}+00$ & $3.8072 \mathrm{E}+00$ & $9.3641 \mathrm{E}-01$ \\
\hline & & Rosenbrock & $1.3566 \mathrm{E}-01$ & $6.4893 \mathrm{E}-02$ & $1.7064 \mathrm{E}-01$ & $3.7665 \mathrm{E}-02$ \\
\hline & & Sphere & $2.8319 \mathrm{E}+00$ & $1.9562 \mathrm{E}+00$ & $4.0622 \mathrm{E}+00$ & 8.5459E-01 \\
\hline & \multirow{5}{*}{15} & Ackley & $7.8990 \mathrm{E}+03$ & $1.1034 \mathrm{E}+04$ & $1.7050 \mathrm{E}+04$ & $4.1550 \mathrm{E}+03$ \\
\hline & & Alpine & $1.1978 \mathrm{E}+04$ & $9.7005 \mathrm{E}+03$ & $1.4333 \mathrm{E}+04$ & $7.6525 \mathrm{E}+03$ \\
\hline & & Rastrigin & $4.9819 \mathrm{E}+00$ & $9.0338 \mathrm{E}+00$ & $1.1208 \mathrm{E}+01$ & $2.4916 \mathrm{E}+00$ \\
\hline & & Rosenbrock & $1.7790 \mathrm{E}-01$ & $1.8475 \mathrm{E}-01$ & $2.7734 \mathrm{E}-01$ & $6.2758 \mathrm{E}-02$ \\
\hline & & Sphere & $7.3874 \mathrm{E}+00$ & $6.0451 \mathrm{E}+00$ & $1.0039 \mathrm{E}+01$ & $2.6722 \mathrm{E}+00$ \\
\hline & \multirow{5}{*}{25} & Ackley & 4.4147E+04 & $4.8260 \mathrm{E}+04$ & $6.8625 \mathrm{E}+04$ & $2.1149 \mathrm{E}+04$ \\
\hline & & Alpine & $2.7758 \mathrm{E}+04$ & $3.7306 \mathrm{E}+04$ & $4.0710 \mathrm{E}+04$ & $2.1629 \mathrm{E}+03$ \\
\hline & & Rastrigin & $2.8679 \mathrm{E}+01$ & $3.5251 \mathrm{E}+01$ & $5.5838 \mathrm{E}+01$ & $1.3696 \mathrm{E}+01$ \\
\hline & & Rosenbrock & 7.8127E-01 & $7.6840 \mathrm{E}-01$ & $1.2919 \mathrm{E}+00$ & $3.5778 \mathrm{E}-01$ \\
\hline & & Sphere & $3.2185 \mathrm{E}+01$ & $3.4547 \mathrm{E}+01$ & $5.2285 \mathrm{E}+01$ & $1.4817 \mathrm{E}+01$ \\
\hline \multirow{15}{*}{75} & \multirow{5}{*}{10} & Ackley & $3.2329 \mathrm{E}+03$ & $1.6534 \mathrm{E}+03$ & $3.7019 \mathrm{E}+03$ & $1.1790 \mathrm{E}+03$ \\
\hline & & Alpine & $2.6275 \mathrm{E}+03$ & $2.9863 \mathrm{E}+03$ & $3.3332 \mathrm{E}+03$ & $3.0911 \mathrm{E}+03$ \\
\hline & & Rastrigin & $3.4479 \mathrm{E}+00$ & $2.6589 \mathrm{E}+00$ & $4.2136 \mathrm{E}+00$ & $2.8323 \mathrm{E}+00$ \\
\hline & & Rosenbrock & $1.2037 \mathrm{E}-01$ & $5.9902 \mathrm{E}-02$ & $1.2761 \mathrm{E}-01$ & $1.0750 \mathrm{E}-01$ \\
\hline & & Sphere & $2.7578 \mathrm{E}+00$ & $2.2675 \mathrm{E}+00$ & $6.5196 \mathrm{E}+00$ & $1.8821 \mathrm{E}+00$ \\
\hline & \multirow{5}{*}{15} & Ackley & $6.4111 \mathrm{E}+03$ & $8.5505 \mathrm{E}+03$ & $1.1051 \mathrm{E}+04$ & $2.8486 \mathrm{E}+03$ \\
\hline & & Alpine & $1.0990 \mathrm{E}+04$ & $1.1736 \mathrm{E}+04$ & $1.2430 \mathrm{E}+04$ & $7.7682 \mathrm{E}+03$ \\
\hline & & Rastrigin & $6.0159 \mathrm{E}+00$ & $6.1844 \mathrm{E}+00$ & $7.9603 \mathrm{E}+00$ & $1.8481 \mathrm{E}+00$ \\
\hline & & Rosenbrock & $1.5541 \mathrm{E}-01$ & $1.2363 \mathrm{E}-01$ & $2.3267 \mathrm{E}-01$ & $5.1811 \mathrm{E}-02$ \\
\hline & & Sphere & $4.8801 \mathrm{E}+00$ & $5.5035 \mathrm{E}+00$ & $9.0839 \mathrm{E}+00$ & $1.9854 \mathrm{E}+00$ \\
\hline & \multirow{5}{*}{25} & Ackley & $3.8895 \mathrm{E}+04$ & $4.5532 \mathrm{E}+04$ & $6.0800 \mathrm{E}+04$ & $1.5344 \mathrm{E}+04$ \\
\hline & & Alpine & $2.3632 \mathrm{E}+03$ & $3.0962 \mathrm{E}+04$ & $4.0510 \mathrm{E}+04$ & $2.0621 \mathrm{E}+04$ \\
\hline & & Rastrigin & $2.6194 \mathrm{E}+01$ & $2.8591 \mathrm{E}+01$ & $3.9536 \mathrm{E}+01$ & $9.0635 \mathrm{E}+00$ \\
\hline & & Rosenbrock & 5.4187E-01 & $7.9861 \mathrm{E}-01$ & $9.8031 \mathrm{E}-01$ & $2.5143 \mathrm{E}-01$ \\
\hline & & Sphere & $2.4697 \mathrm{E}+01$ & $2.6905 \mathrm{E}+01$ & $3.7949 \mathrm{E}+01$ & $1.0481 \mathrm{E}+01$ \\
\hline \multirow{15}{*}{100} & \multirow{5}{*}{10} & Ackley & $2.3993 \mathrm{E}+03$ & $2.3529 \mathrm{E}+03$ & $4.5123 \mathrm{E}+03$ & $2.4679 \mathrm{E}+03$ \\
\hline & & Alpine & $3.8224 \mathrm{E}+03$ & $3.5482 \mathrm{E}+03$ & $3.1984 \mathrm{E}+03$ & $2.1321 \mathrm{E}+03$ \\
\hline & & Rastrigin & $1.9674 \mathrm{E}+00$ & $2.0647 \mathrm{E}+00$ & $3.9164 \mathrm{E}+00$ & $1.9696 \mathrm{E}+00$ \\
\hline & & Rosenbrock & $9.8795 \mathrm{E}-02$ & $5.0247 \mathrm{E}-02$ & $1.0518 \mathrm{E}-01$ & 7.1418E-02 \\
\hline & & Sphere & $2.7045 \mathrm{E}+00$ & $1.6557 \mathrm{E}+00$ & $4.7904 \mathrm{E}+00$ & $2.9823 \mathrm{E}+00$ \\
\hline & \multirow{5}{*}{15} & Ackley & $5.9991 \mathrm{E}+03$ & $6.3815 \mathrm{E}+03$ & $1.1584 \mathrm{E}+04$ & $2.0854 \mathrm{E}+03$ \\
\hline & & Alpine & $1.1210 \mathrm{E}+04$ & $1.0753 \mathrm{E}+04$ & $1.0897 \mathrm{E}+04$ & $7.3446 \mathrm{E}+03$ \\
\hline & & Rastrigin & $4.2407 \mathrm{E}+00$ & $4.0977 \mathrm{E}+00$ & $6.9168 \mathrm{E}+00$ & $1.4860 \mathrm{E}+00$ \\
\hline & & Rosenbrock & $1.3515 \mathrm{E}-01$ & $1.6768 \mathrm{E}-01$ & $2.4625 \mathrm{E}-01$ & $3.9636 \mathrm{E}-02$ \\
\hline & & Sphere & $5.1469 \mathrm{E}+00$ & $5.5297 \mathrm{E}+00$ & $8.2649 \mathrm{E}+00$ & $1.6979 \mathrm{E}+00$ \\
\hline & \multirow{5}{*}{25} & Ackley & $3.2497 \mathrm{E}+04$ & $3.3093 \mathrm{E}+04$ & $4.6154 \mathrm{E}+04$ & $1.2714 \mathrm{E}+04$ \\
\hline & & Alpine & $2.9151 \mathrm{E}+04$ & $4.0348 \mathrm{E}+04$ & $3.9757 \mathrm{E}+04$ & $1.5633 \mathrm{E}+03$ \\
\hline & & Rastrigin & $1.8973 \mathrm{E}+01$ & $2.1542 \mathrm{E}+01$ & $3.4920 \mathrm{E}+01$ & $9.0575 \mathrm{E}+00$ \\
\hline & & Rosenbrock & $5.1319 \mathrm{E}-01$ & $6.9884 \mathrm{E}-01$ & $7.5790 \mathrm{E}-01$ & $2.2551 \mathrm{E}-01$ \\
\hline & & Sphere & $1.8716 \mathrm{E}+01$ & $1.7228 \mathrm{E}+01$ & $3.0362 \mathrm{E}+01$ & $8.9174 \mathrm{E}+00$ \\
\hline
\end{tabular}


Comparison of PSOs with Variance
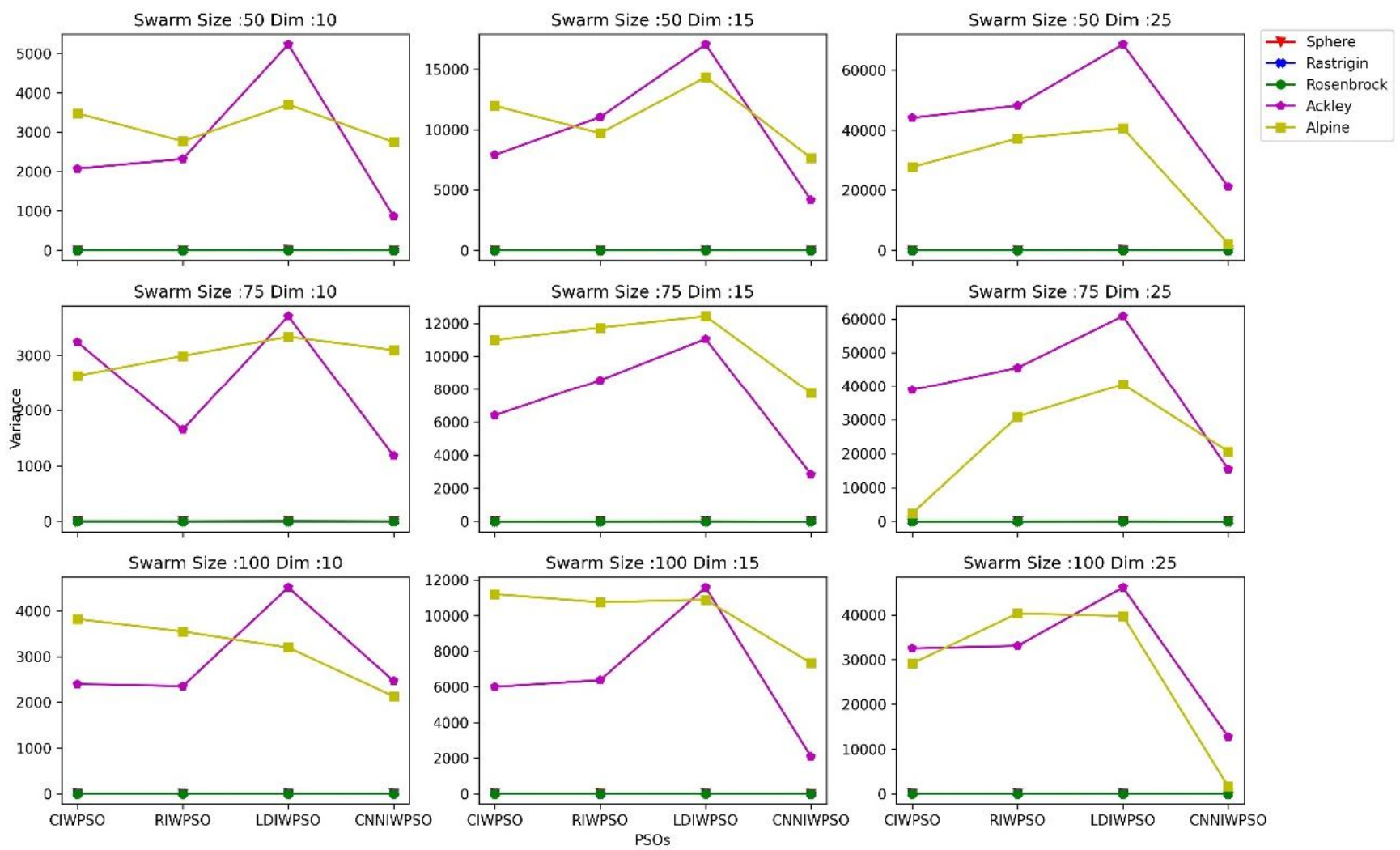

Figure 6: Variance computed for the swarm size of 50, 75 and 100 with dimensions 10, 15 and 25 
Y.V.R. Naga Pawan et al., International Journal of Emerging Trends in Engineering Research, 8(9), September 2020, 6111 - 6130

Table 5: Computed Standard Deviation for PSOs with respect different Swarm Sizes and Dimensions. (Figure 7)

\begin{tabular}{|c|c|c|c|c|c|c|}
\hline \multirow{2}{*}{ Swarm Size } & \multirow{2}{*}{ Dimension } & \multirow{2}{*}{ BMF } & \multicolumn{4}{|c|}{ PSOs } \\
\hline & & & CIWPSO & RIWPSO & LDIWPSO & CNNIWPSO \\
\hline \multirow{15}{*}{50} & \multirow{5}{*}{10} & Ackley & $4.5543 \mathrm{E}+01$ & $4.8178 \mathrm{E}+01$ & $7.2306 \mathrm{E}+01$ & $2.9313 \mathrm{E}+01$ \\
\hline & & Alpine & $5.8974 \mathrm{E}+01$ & $5.2637 \mathrm{E}+01$ & $6.0842 \mathrm{E}+01$ & $5.2430 \mathrm{E}+01$ \\
\hline & & Rastrigin & $1.5985 \mathrm{E}+00$ & $1.5784 \mathrm{E}+00$ & $1.9512 \mathrm{E}+00$ & $9.6769 \mathrm{E}-01$ \\
\hline & & Rosenbrock & $3.6832 \mathrm{E}-01$ & $2.5474 \mathrm{E}-01$ & $4.1309 \mathrm{E}-01$ & $1.9408 \mathrm{E}-01$ \\
\hline & & Sphere & $1.6828 \mathrm{E}+00$ & $1.3986 \mathrm{E}+00$ & $2.0155 \mathrm{E}+00$ & $9.2444 \mathrm{E}-01$ \\
\hline & \multirow{5}{*}{15} & Ackley & $8.8876 \mathrm{E}+01$ & $1.0504 \mathrm{E}+02$ & $1.3058 \mathrm{E}+02$ & $6.4460 \mathrm{E}+01$ \\
\hline & & Alpine & $1.0944 \mathrm{E}+02$ & $9.8491 \mathrm{E}+01$ & $1.1972 \mathrm{E}+02$ & $8.7478 \mathrm{E}+01$ \\
\hline & & Rastrigin & $2.2320 \mathrm{E}+00$ & $3.0056 \mathrm{E}+00$ & $3.3478 \mathrm{E}+00$ & $1.5785 \mathrm{E}+00$ \\
\hline & & Rosenbrock & $4.2179 \mathrm{E}-01$ & $4.2982 \mathrm{E}-01$ & $5.2663 \mathrm{E}-01$ & $2.5052 \mathrm{E}-01$ \\
\hline & & Sphere & $2.7180 \mathrm{E}+00$ & $2.4587 \mathrm{E}+00$ & $3.1684 \mathrm{E}+00$ & $1.6347 \mathrm{E}+00$ \\
\hline & \multirow{5}{*}{25} & Ackley & $2.1011 \mathrm{E}+02$ & $2.1968 \mathrm{E}+02$ & $2.6196 \mathrm{E}+02$ & $1.4543 \mathrm{E}+02$ \\
\hline & & Alpine & $1.6661 \mathrm{E}+02$ & $1.9315 \mathrm{E}+02$ & $2.0177 \mathrm{E}+02$ & $4.6507 \mathrm{E}+01$ \\
\hline & & Rastrigin & $5.3553 \mathrm{E}+00$ & $5.9373 \mathrm{E}+00$ & $7.4725 \mathrm{E}+00$ & $3.7008 \mathrm{E}+00$ \\
\hline & & Rosenbrock & $8.8390 \mathrm{E}-01$ & $8.7659 \mathrm{E}-01$ & $1.1366 \mathrm{E}+00$ & $5.9815 \mathrm{E}-01$ \\
\hline & & Sphere & $5.6732 \mathrm{E}+00$ & $5.8777 \mathrm{E}+00$ & $7.2308 \mathrm{E}+00$ & $3.8493 \mathrm{E}+00$ \\
\hline \multirow{15}{*}{75} & \multirow{5}{*}{10} & Ackley & $5.6859 \mathrm{E}+01$ & $4.0661 \mathrm{E}+01$ & $6.0843 \mathrm{E}+01$ & $3.4336 \mathrm{E}+01$ \\
\hline & & Alpine & $5.1259 \mathrm{E}+01$ & $5.4647 \mathrm{E}+01$ & $5.7734 \mathrm{E}+01$ & $5.5598 \mathrm{E}+01$ \\
\hline & & Rastrigin & $1.8569 \mathrm{E}+00$ & $1.6306 \mathrm{E}+00$ & $2.0527 \mathrm{E}+00$ & $1.6830 \mathrm{E}+00$ \\
\hline & & Rosenbrock & $3.4694 \mathrm{E}-01$ & $2.4475 \mathrm{E}-01$ & $3.5722 \mathrm{E}-01$ & $3.2787 \mathrm{E}-01$ \\
\hline & & Sphere & $1.6607 \mathrm{E}+00$ & $1.5058 \mathrm{E}+00$ & $2.5534 \mathrm{E}+00$ & $1.3719 \mathrm{E}+00$ \\
\hline & \multirow{5}{*}{15} & Ackley & $8.0070 \mathrm{E}+01$ & $9.2469 \mathrm{E}+01$ & $1.0512 \mathrm{E}+02$ & $5.3373 \mathrm{E}+01$ \\
\hline & & Alpine & $1.0483 \mathrm{E}+02$ & $1.0833 \mathrm{E}+02$ & $1.1149 \mathrm{E}+02$ & $8.8137 \mathrm{E}+01$ \\
\hline & & Rastrigin & $2.4527 \mathrm{E}+00$ & $2.4869 \mathrm{E}+00$ & $2.8214 \mathrm{E}+00$ & $1.3594 \mathrm{E}+00$ \\
\hline & & Rosenbrock & $3.9422 \mathrm{E}-01$ & $3.5161 \mathrm{E}-01$ & $4.8236 \mathrm{E}-01$ & $2.2762 \mathrm{E}-01$ \\
\hline & & Sphere & $2.2091 \mathrm{E}+00$ & $2.3459 \mathrm{E}+00$ & $3.0139 \mathrm{E}+00$ & $1.4090 \mathrm{E}+00$ \\
\hline & \multirow{5}{*}{25} & Ackley & $1.9722 \mathrm{E}+02$ & $2.1338 \mathrm{E}+02$ & $2.4658 \mathrm{E}+02$ & $1.2387 \mathrm{E}+02$ \\
\hline & & Alpine & $4.8613 \mathrm{E}+01$ & $1.7596 \mathrm{E}+02$ & $2.0127 \mathrm{E}+02$ & $1.4360 \mathrm{E}+02$ \\
\hline & & Rastrigin & $5.1180 \mathrm{E}+00$ & $5.3471 \mathrm{E}+00$ & $6.2878 \mathrm{E}+00$ & $3.0106 \mathrm{E}+00$ \\
\hline & & Rosenbrock & 7.3612E-01 & $8.9365 \mathrm{E}-01$ & $9.9011 \mathrm{E}-01$ & $5.0143 \mathrm{E}-01$ \\
\hline & & Sphere & $4.9696 \mathrm{E}+00$ & $5.1870 \mathrm{E}+00$ & $6.1603 \mathrm{E}+00$ & $3.2374 \mathrm{E}+00$ \\
\hline \multirow{15}{*}{100} & \multirow{5}{*}{10} & Ackley & $4.8983 \mathrm{E}+01$ & $4.8507 \mathrm{E}+01$ & $6.7174 \mathrm{E}+01$ & $4.9678 \mathrm{E}+01$ \\
\hline & & Alpine & $6.1825 \mathrm{E}+01$ & $5.9567 \mathrm{E}+01$ & $5.6554 \mathrm{E}+01$ & $4.6174 \mathrm{E}+01$ \\
\hline & & Rastrigin & $1.4026 \mathrm{E}+00$ & $1.4369 \mathrm{E}+00$ & $1.9790 \mathrm{E}+00$ & $1.4034 \mathrm{E}+00$ \\
\hline & & Rosenbrock & $3.1432 \mathrm{E}-01$ & $2.2416 \mathrm{E}-01$ & $3.2432 \mathrm{E}-01$ & $2.6724 \mathrm{E}-01$ \\
\hline & & Sphere & $1.6445 E+00$ & $1.2868 \mathrm{E}+00$ & $2.1887 \mathrm{E}+00$ & $1.7269 \mathrm{E}+00$ \\
\hline & \multirow{5}{*}{15} & Ackley & $7.7454 \mathrm{E}+01$ & $7.9884 \mathrm{E}+01$ & $1.0763 \mathrm{E}+02$ & $4.5666 \mathrm{E}+01$ \\
\hline & & Alpine & $1.0588 \mathrm{E}+02$ & $1.0370 \mathrm{E}+02$ & $1.0439 \mathrm{E}+02$ & $8.5701 \mathrm{E}+01$ \\
\hline & & Rastrigin & $2.0593 \mathrm{E}+00$ & $2.0243 \mathrm{E}+00$ & $2.6300 \mathrm{E}+00$ & $1.2190 \mathrm{E}+00$ \\
\hline & & Rosenbrock & $3.6763 \mathrm{E}-01$ & $4.0949 \mathrm{E}-01$ & $4.9624 \mathrm{E}-01$ & $1.9909 \mathrm{E}-01$ \\
\hline & & Sphere & $2.2687 \mathrm{E}+00$ & $2.3515 \mathrm{E}+00$ & $2.8749 \mathrm{E}+00$ & $1.3030 \mathrm{E}+00$ \\
\hline & \multirow{5}{*}{25} & Ackley & $1.8027 \mathrm{E}+02$ & $1.8191 \mathrm{E}+02$ & $2.1483 \mathrm{E}+02$ & $1.1276 \mathrm{E}+02$ \\
\hline & & Alpine & $1.7074 \mathrm{E}+02$ & $2.0087 \mathrm{E}+02$ & $1.9939 \mathrm{E}+02$ & $3.9539 \mathrm{E}+01$ \\
\hline & & Rastrigin & $4.3558 \mathrm{E}+00$ & $4.6413 \mathrm{E}+00$ & $5.9093 \mathrm{E}+00$ & $3.0096 \mathrm{E}+00$ \\
\hline & & Rosenbrock & 7.1637E-01 & 8.3597E-01 & $8.7057 \mathrm{E}-01$ & $4.7488 \mathrm{E}-01$ \\
\hline & & Sphere & $4.3262 \mathrm{E}+00$ & $4.1507 \mathrm{E}+00$ & $5.5101 \mathrm{E}+00$ & $2.9862 \mathrm{E}+00$ \\
\hline
\end{tabular}


Y.V.R. Naga Pawan et al., International Journal of Emerging Trends in Engineering Research, 8(9), September 2020, 6111 - 6130

Comparison of PSOs with Standard Deviation
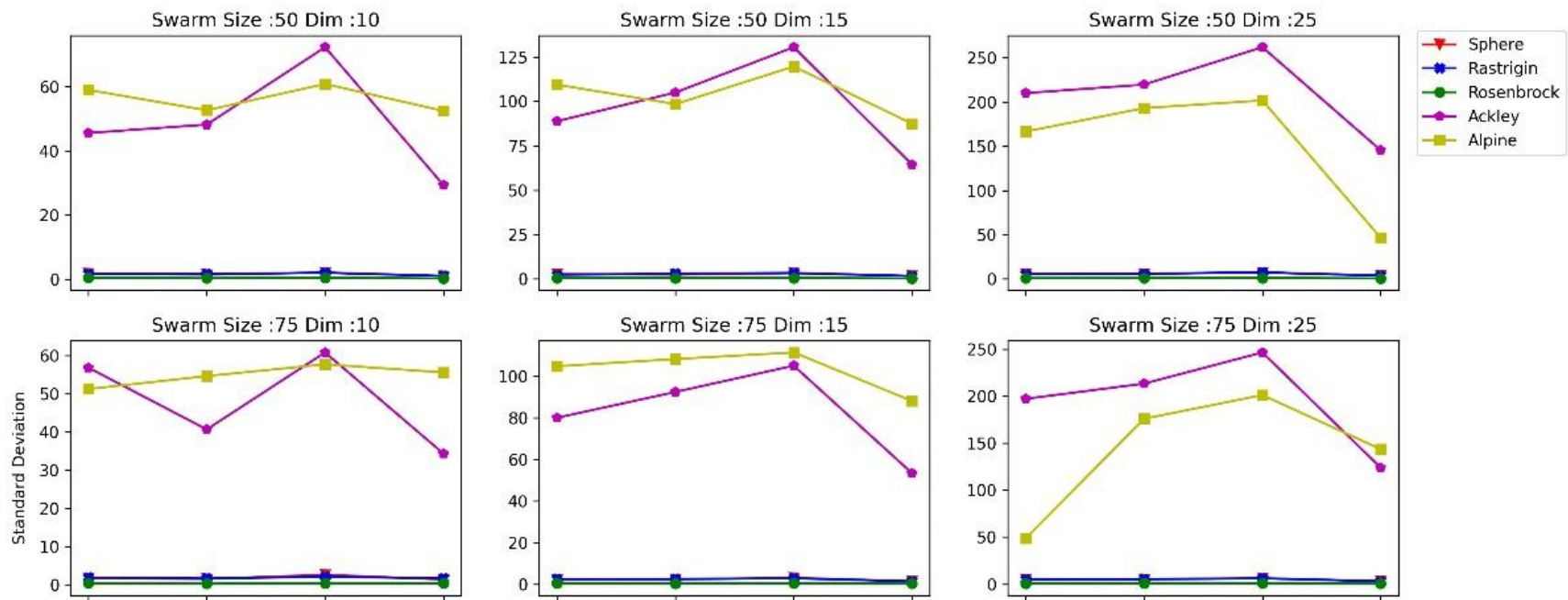

Swarm Size :75 Dim :15

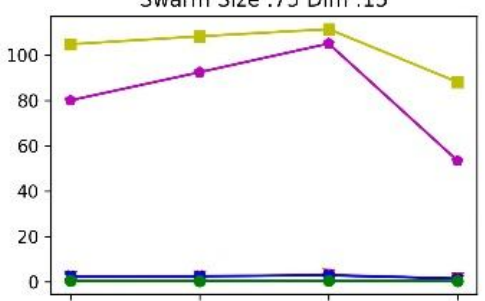

Swarm Size :75 Dim :25
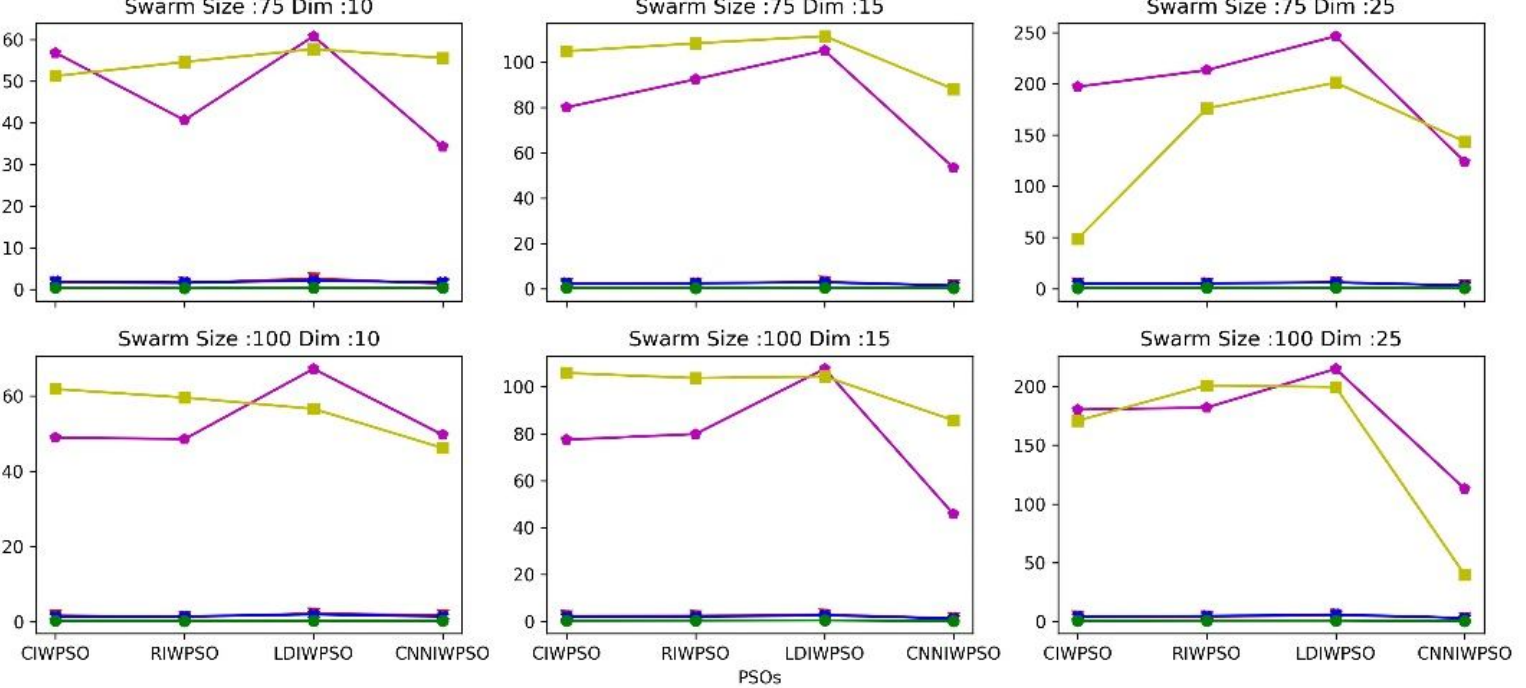

Figure 7: Standard Deviation computed for the swarm size of 50, 75 and 100 with dimensions 10, 15 and 25

Table 4, Table 5, Figure 6 Figure 7 shows that the CNNIWPSO has the flair in terms of variance and standard deviation with increasing swarm sizes and dimensions. 
Y.V.R. Naga Pawan et al., International Journal of Emerging Trends in Engineering Research, 8(9), September 2020, 6111 - 6130

Table 6: Computed Mean Iterations for PSOs with respect different Swarm Sizes and Dimensions. (Figure 8)

\begin{tabular}{|c|c|c|c|c|c|c|}
\hline \multirow{2}{*}{ Swarm Size } & \multirow{2}{*}{ Dimension } & \multirow{2}{*}{ BMF } & \multicolumn{4}{|c|}{ PSOs } \\
\hline & & & CIWPSO & RIWPSO & LDIWPSO & CNNIWPSO \\
\hline \multirow{15}{*}{50} & \multirow{5}{*}{10} & Ackley & $9.8360 \mathrm{E}+02$ & $9.2550 \mathrm{E}+02$ & $9.5070 \mathrm{E}+02$ & $1.0000 \mathrm{E}+03$ \\
\hline & & Alpine & $5.0000 \mathrm{E}+00$ & $4.9000 \mathrm{E}+00$ & $5.1000 \mathrm{E}+00$ & $5.9333 \mathrm{E}+00$ \\
\hline & & Rastrigin & $4.2950 \mathrm{E}+02$ & $6.6420 \mathrm{E}+02$ & $7.2250 \mathrm{E}+02$ & $5.7247 \mathrm{E}+02$ \\
\hline & & Rosenbrock & $3.1850 \mathrm{E}+02$ & $6.4790 \mathrm{E}+02$ & $4.6320 \mathrm{E}+02$ & $3.5520 \mathrm{E}+02$ \\
\hline & & Sphere & $5.7760 \mathrm{E}+02$ & $8.8540 \mathrm{E}+02$ & $6.7550 \mathrm{E}+02$ & $6.8513 \mathrm{E}+02$ \\
\hline & \multirow{5}{*}{15} & Ackley & $1.0000 \mathrm{E}+03$ & $1.0000 \mathrm{E}+03$ & $1.0000 \mathrm{E}+03$ & $1.0000 \mathrm{E}+03$ \\
\hline & & Alpine & $6.7000 \mathrm{E}+00$ & $6.6000 \mathrm{E}+00$ & $5.4000 \mathrm{E}+00$ & $1.2200 \mathrm{E}+01$ \\
\hline & & Rastrigin & $1.0000 \mathrm{E}+03$ & $1.0000 \mathrm{E}+03$ & $1.0000 \mathrm{E}+03$ & $1.0000 \mathrm{E}+03$ \\
\hline & & Rosenbrock & $1.0000 \mathrm{E}+03$ & $1.0000 \mathrm{E}+03$ & $1.0000 \mathrm{E}+03$ & $1.0000 \mathrm{E}+03$ \\
\hline & & Sphere & $1.0000 \mathrm{E}+03$ & $1.0000 \mathrm{E}+03$ & $1.0000 \mathrm{E}+03$ & $1.0000 \mathrm{E}+03$ \\
\hline & \multirow{5}{*}{25} & Ackley & $1.0000 \mathrm{E}+03$ & $1.0000 \mathrm{E}+03$ & $1.0000 \mathrm{E}+03$ & $1.0000 \mathrm{E}+03$ \\
\hline & & Alpine & $1.0700 \mathrm{E}+01$ & $8.2000 \mathrm{E}+00$ & $8.5000 \mathrm{E}+00$ & $1.4687 \mathrm{E}+02$ \\
\hline & & Rastrigin & $1.0000 \mathrm{E}+03$ & $1.0000 \mathrm{E}+03$ & $1.0000 \mathrm{E}+03$ & $1.0000 \mathrm{E}+03$ \\
\hline & & Rosenbrock & $1.0000 \mathrm{E}+03$ & $1.0000 \mathrm{E}+03$ & $1.0000 \mathrm{E}+03$ & $1.0000 \mathrm{E}+03$ \\
\hline & & Sphere & $1.0000 \mathrm{E}+03$ & $1.0000 \mathrm{E}+03$ & $1.0000 \mathrm{E}+03$ & $1.0000 \mathrm{E}+03$ \\
\hline \multirow{15}{*}{75} & \multirow{5}{*}{10} & Ackley & $5.8230 \mathrm{E}+02$ & $6.7690 \mathrm{E}+02$ & $7.8380 \mathrm{E}+02$ & $4.5020 \mathrm{E}+02$ \\
\hline & & Alpine & $5.8000 \mathrm{E}+00$ & $4.0000 \mathrm{E}+00$ & $4.1000 \mathrm{E}+00$ & $5.0667 \mathrm{E}+00$ \\
\hline & & Rastrigin & $3.3310 \mathrm{E}+02$ & $4.7770 \mathrm{E}+02$ & $4.2040 \mathrm{E}+02$ & $1.5540 \mathrm{E}+02$ \\
\hline & & Rosenbrock & $2.3050 \mathrm{E}+02$ & $4.7880 \mathrm{E}+02$ & $3.9630 \mathrm{E}+02$ & $1.1453 \mathrm{E}+02$ \\
\hline & & Sphere & $3.8000 \mathrm{E}+02$ & $4.3010 \mathrm{E}+02$ & $4.2160 \mathrm{E}+02$ & $1.9653 \mathrm{E}+02$ \\
\hline & \multirow{5}{*}{15} & Ackley & $1.0000 \mathrm{E}+03$ & $1.0000 \mathrm{E}+03$ & $1.0000 \mathrm{E}+03$ & $1.0000 \mathrm{E}+03$ \\
\hline & & Alpine & $6.5000 \mathrm{E}+00$ & $5.8000 \mathrm{E}+00$ & $5.1000 \mathrm{E}+00$ & $7.1333 \mathrm{E}+00$ \\
\hline & & Rastrigin & $9.1500 \mathrm{E}+02$ & $1.0000 \mathrm{E}+03$ & $9.4610 \mathrm{E}+02$ & $1.0000 \mathrm{E}+03$ \\
\hline & & Rosenbrock & $7.8950 \mathrm{E}+02$ & $1.0000 \mathrm{E}+03$ & $8.8900 \mathrm{E}+02$ & $9.4580 \mathrm{E}+02$ \\
\hline & & Sphere & $9.5170 \mathrm{E}+02$ & $1.0000 \mathrm{E}+03$ & $1.0000 \mathrm{E}+03$ & $1.0000 \mathrm{E}+03$ \\
\hline & \multirow{5}{*}{25} & Ackley & $1.0000 \mathrm{E}+03$ & $1.0000 \mathrm{E}+03$ & $1.0000 \mathrm{E}+03$ & $1.0000 \mathrm{E}+03$ \\
\hline & & Alpine & $1.0610 \mathrm{E}+02$ & $8.7000 \mathrm{E}+00$ & $7.0000 \mathrm{E}+00$ & $1.4933 \mathrm{E}+01$ \\
\hline & & Rastrigin & $1.0000 \mathrm{E}+03$ & $1.0000 \mathrm{E}+03$ & $1.0000 \mathrm{E}+03$ & $1.0000 \mathrm{E}+03$ \\
\hline & & Rosenbrock & $1.0000 \mathrm{E}+03$ & $1.0000 \mathrm{E}+03$ & $1.0000 \mathrm{E}+03$ & $1.0000 \mathrm{E}+03$ \\
\hline & & Sphere & $1.0000 \mathrm{E}+03$ & $1.0000 \mathrm{E}+03$ & $1.0000 \mathrm{E}+03$ & $1.0000 \mathrm{E}+03$ \\
\hline \multirow{15}{*}{100} & \multirow{5}{*}{10} & Ackley & $4.9970 \mathrm{E}+02$ & $5.9840 \mathrm{E}+02$ & $5.1280 \mathrm{E}+02$ & $1.7227 \mathrm{E}+02$ \\
\hline & & Alpine & $3.8000 \mathrm{E}+00$ & $4.1000 \mathrm{E}+00$ & $4.0000 \mathrm{E}+00$ & $3.9333 \mathrm{E}+00$ \\
\hline & & Rastrigin & $3.4610 \mathrm{E}+02$ & $5.3120 \mathrm{E}+02$ & $4.0780 \mathrm{E}+02$ & $1.2327 \mathrm{E}+02$ \\
\hline & & Rosenbrock & $2.0200 \mathrm{E}+02$ & $4.8650 \mathrm{E}+02$ & $3.7890 \mathrm{E}+02$ & $9.4067 \mathrm{E}+01$ \\
\hline & & Sphere & $2.5060 \mathrm{E}+02$ & $6.1100 \mathrm{E}+02$ & $4.0150 \mathrm{E}+02$ & $1.1447 \mathrm{E}+02$ \\
\hline & \multirow{5}{*}{15} & Ackley & $9.8310 \mathrm{E}+02$ & $1.0000 \mathrm{E}+03$ & $1.0000 \mathrm{E}+03$ & $1.0000 \mathrm{E}+03$ \\
\hline & & Alpine & $4.8000 \mathrm{E}+00$ & $5.2000 \mathrm{E}+00$ & $4.8000 \mathrm{E}+00$ & $7.4000 \mathrm{E}+00$ \\
\hline & & Rastrigin & $9.3740 \mathrm{E}+02$ & $1.0000 \mathrm{E}+03$ & $9.4700 \mathrm{E}+02$ & $9.0213 E+02$ \\
\hline & & Rosenbrock & $6.6340 \mathrm{E}+02$ & $5.9040 \mathrm{E}+02$ & $7.8470 \mathrm{E}+02$ & $8.9360 \mathrm{E}+02$ \\
\hline & & Sphere & $8.2110 \mathrm{E}+02$ & $9.8140 \mathrm{E}+02$ & $9.4450 \mathrm{E}+02$ & $1.0000 \mathrm{E}+03$ \\
\hline & \multirow{5}{*}{25} & Ackley & $1.0000 \mathrm{E}+03$ & $1.0000 \mathrm{E}+03$ & $1.0000 \mathrm{E}+03$ & $1.0000 \mathrm{E}+03$ \\
\hline & & Alpine & $9.6000 \mathrm{E}+00$ & $5.3000 \mathrm{E}+00$ & $5.7000 \mathrm{E}+00$ & $1.4040 \mathrm{E}+02$ \\
\hline & & Rastrigin & $1.0000 \mathrm{E}+03$ & $1.0000 \mathrm{E}+03$ & $1.0000 \mathrm{E}+03$ & $1.0000 \mathrm{E}+03$ \\
\hline & & Rosenbrock & $1.0000 \mathrm{E}+03$ & $1.0000 \mathrm{E}+03$ & $1.0000 \mathrm{E}+03$ & $1.0000 \mathrm{E}+03$ \\
\hline & & Sphere & $1.0000 \mathrm{E}+03$ & $1.0000 \mathrm{E}+03$ & $1.0000 \mathrm{E}+03$ & $1.0000 \mathrm{E}+03$ \\
\hline
\end{tabular}


Comparison of PSOs with Mean Iterations
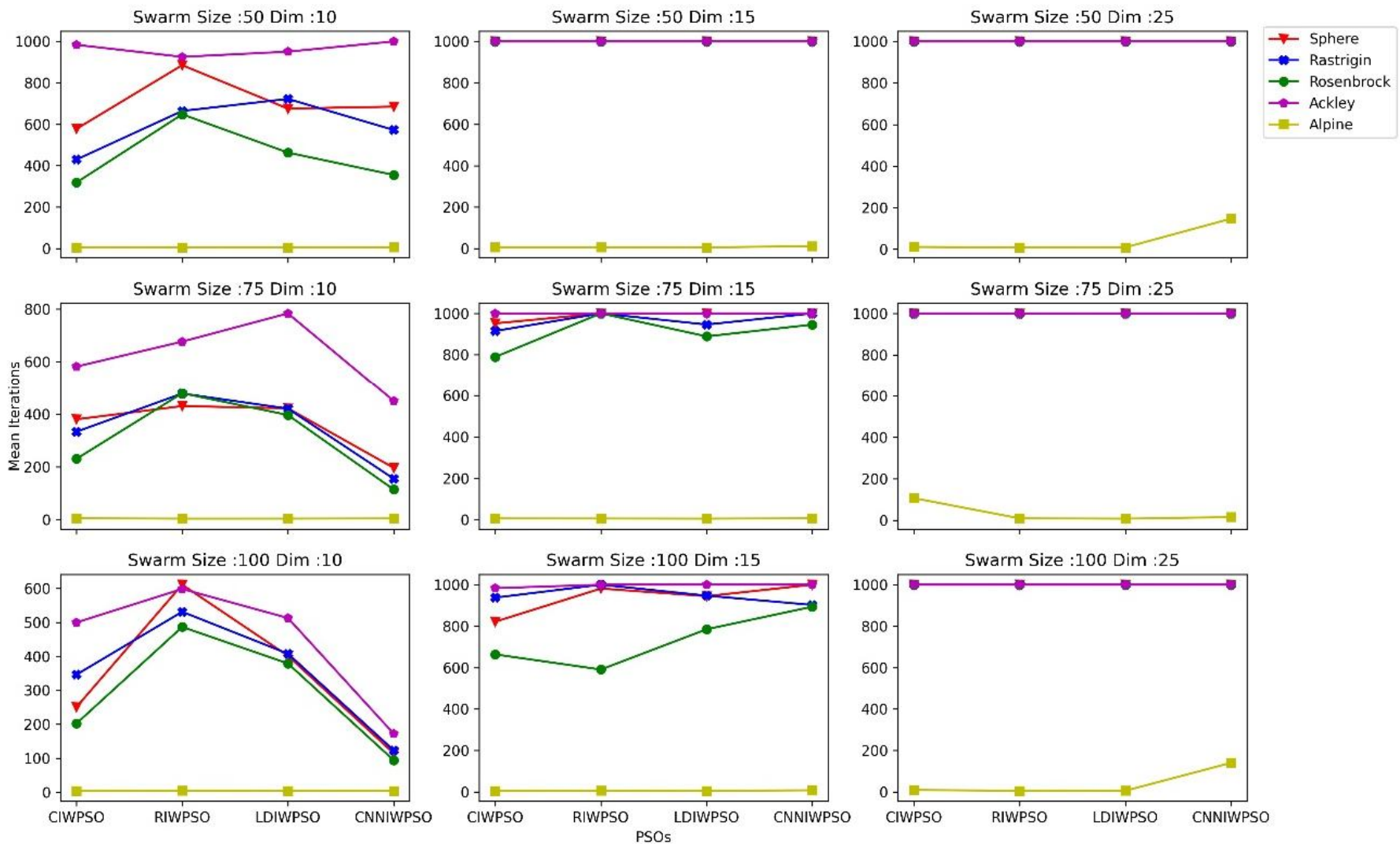

Figure 8: Mean Iterations computed for the swarm size of 50, 75 and 100 with dimensions 10, 15 and 25

From the Table 6 and Figure 8, the mean iterations for CNNIWPSO are better when compared to other models for the Ackley and Sphere benchmark function with Swarm size 100 and dimension 10. Incase of Alpine benchmark function,
CNNIWPSO is superior with increasing swarm sizes and dimensions. Analogously, CNNIWPSO given better results with Rosenbrock and Rastrigin benchmark function. 
Y.V.R. Naga Pawan et al., International Journal of Emerging Trends in Engineering Research, 8(9), September 2020, 6111 - 6130

Table 7: Computed Mean Time (in seconds) for PSOs with respect different Swarm Sizes and Dimensions. (Figure 9)

\begin{tabular}{|c|c|c|c|c|c|c|}
\hline \multirow{2}{*}{ Swarm Size } & \multirow{2}{*}{ Dimension } & \multirow{2}{*}{ BMF } & \multicolumn{4}{|c|}{ PSOs } \\
\hline & & & CIWPSO & RIWPSO & LDIWPSO & CNNIWPSO \\
\hline \multirow{15}{*}{50} & \multirow{5}{*}{10} & Ackley & $2.0295 \mathrm{E}+00$ & $1.9307 \mathrm{E}+00$ & $2.0037 \mathrm{E}+00$ & $6.9546 \mathrm{E}+00$ \\
\hline & & Alpine & $1.0961 \mathrm{E}-02$ & $1.0921 \mathrm{E}-02$ & $1.1749 \mathrm{E}-02$ & 4.3107E-02 \\
\hline & & Rastrigin & 8.8939E-01 & $1.3824 \mathrm{E}+00$ & $1.5142 \mathrm{E}+00$ & $3.8330 \mathrm{E}+00$ \\
\hline & & Rosenbrock & $6.5593 \mathrm{E}-01$ & $1.3430 \mathrm{E}+00$ & $9.6360 \mathrm{E}-01$ & $2.3669 \mathrm{E}+00$ \\
\hline & & Sphere & $1.1888 \mathrm{E}+00$ & $1.8592 \mathrm{E}+00$ & $1.4097 \mathrm{E}+00$ & $4.5820 \mathrm{E}+00$ \\
\hline & \multirow{5}{*}{15} & Ackley & $2.6649 \mathrm{E}+00$ & $2.6728 \mathrm{E}+00$ & $2.7205 \mathrm{E}+00$ & $8.6142 \mathrm{E}+00$ \\
\hline & & Alpine & $2.0041 \mathrm{E}-02$ & $1.9509 \mathrm{E}-02$ & $1.6608 \mathrm{E}-02$ & $1.0806 \mathrm{E}-01$ \\
\hline & & Rastrigin & $2.6619 \mathrm{E}+00$ & $2.6897 \mathrm{E}+00$ & $2.7002 \mathrm{E}+00$ & $9.4174 \mathrm{E}+00$ \\
\hline & & Rosenbrock & $2.6658 \mathrm{E}+00$ & $2.6761 \mathrm{E}+00$ & $2.7159 \mathrm{E}+00$ & $9.1439 \mathrm{E}+00$ \\
\hline & & Sphere & $2.6677 \mathrm{E}+00$ & $2.6917 \mathrm{E}+00$ & $2.6823 \mathrm{E}+00$ & $8.6708 \mathrm{E}+00$ \\
\hline & \multirow{5}{*}{25} & Ackley & $3.8367 \mathrm{E}+00$ & $3.8808 \mathrm{E}+00$ & $3.8718 \mathrm{E}+00$ & $1.3050 \mathrm{E}+02$ \\
\hline & & Alpine & $4.3331 \mathrm{E}-02$ & $3.3114 \mathrm{E}-02$ & $3.5782 \mathrm{E}-02$ & $1.8389 \mathrm{E}+00$ \\
\hline & & Rastrigin & $3.8511 \mathrm{E}+00$ & $3.8603 \mathrm{E}+00$ & $3.8848 \mathrm{E}+00$ & $1.3306 \mathrm{E}+01$ \\
\hline & & Rosenbrock & $3.8531 \mathrm{E}+00$ & $3.8625 \mathrm{E}+00$ & $3.8789 \mathrm{E}+00$ & $1.2913 \mathrm{E}+01$ \\
\hline & & Sphere & $3.8381 \mathrm{E}+00$ & $3.8649 \mathrm{E}+00$ & $3.8781 \mathrm{E}+00$ & $1.3015 \mathrm{E}+01$ \\
\hline \multirow{15}{*}{75} & \multirow{5}{*}{10} & Ackley & $1.7874 \mathrm{E}+00$ & $2.1270 \mathrm{E}+00$ & $2.4482 \mathrm{E}+00$ & $4.4598 \mathrm{E}+00$ \\
\hline & & Alpine & $2.1256 \mathrm{E}-02$ & $1.4100 \mathrm{E}-02$ & $1.3580 \mathrm{E}-02$ & $5.2345 \mathrm{E}-02$ \\
\hline & & Rastrigin & $1.0236 \mathrm{E}+00$ & $1.4727 \mathrm{E}+00$ & $1.2995 \mathrm{E}+00$ & $1.5286 \mathrm{E}+00$ \\
\hline & & Rosenbrock & $7.0779 \mathrm{E}-01$ & $1.4837 \mathrm{E}+00$ & $1.2172 \mathrm{E}+00$ & $1.0991 \mathrm{E}+00$ \\
\hline & & Sphere & $1.1695 \mathrm{E}+00$ & $1.3314 \mathrm{E}+00$ & $1.2961 \mathrm{E}+00$ & $1.8666 \mathrm{E}+00$ \\
\hline & \multirow{5}{*}{15} & Ackley & $3.9970 \mathrm{E}+00$ & $3.9939 \mathrm{E}+00$ & $3.9978 \mathrm{E}+00$ & $1.2702 \mathrm{E}+01$ \\
\hline & & Alpine & $2.8363 \mathrm{E}-02$ & $2.6107 \mathrm{E}-02$ & $2.2794 \mathrm{E}-02$ & 9.0737E-02 \\
\hline & & Rastrigin & $3.6478 \mathrm{E}+00$ & $3.9483 \mathrm{E}+00$ & $3.7910 \mathrm{E}+00$ & $1.2878 \mathrm{E}+01$ \\
\hline & & Rosenbrock & $3.1362 \mathrm{E}+00$ & $3.9638 \mathrm{E}+00$ & $3.6008 \mathrm{E}+00$ & $1.2001 \mathrm{E}+01$ \\
\hline & & Sphere & $3.7904 \mathrm{E}+00$ & $3.9833 \mathrm{E}+00$ & $3.9956 \mathrm{E}+00$ & $1.2969 \mathrm{E}+01$ \\
\hline & \multirow{5}{*}{25} & Ackley & $5.7376 \mathrm{E}+00$ & $5.7569 \mathrm{E}+00$ & $5.7887 \mathrm{E}+00$ & $1.9124 \mathrm{E}+01$ \\
\hline & & Alpine & $6.0931 \mathrm{E}-01$ & $5.2966 \mathrm{E}-02$ & $4.3281 \mathrm{E}-02$ & 2.7859E-01 \\
\hline & & Rastrigin & $5.7585 \mathrm{E}+00$ & $5.7611 \mathrm{E}+00$ & $5.7864 \mathrm{E}+00$ & $1.9204 \mathrm{E}+01$ \\
\hline & & Rosenbrock & $5.7413 \mathrm{E}+00$ & $5.7805 \mathrm{E}+00$ & $5.7798 \mathrm{E}+00$ & $2.5188 \mathrm{E}+01$ \\
\hline & & Sphere & $5.7431 \mathrm{E}+00$ & $5.7654 \mathrm{E}+00$ & $5.7950 \mathrm{E}+00$ & $1.8553 \mathrm{E}+01$ \\
\hline \multirow{15}{*}{100} & \multirow{5}{*}{10} & Ackley & $2.0458 \mathrm{E}+00$ & $2.4620 \mathrm{E}+00$ & $2.1090 \mathrm{E}+00$ & $2.1613 \mathrm{E}+00$ \\
\hline & & Alpine & $1.7542 \mathrm{E}-02$ & $1.9902 \mathrm{E}-02$ & $1.7649 \mathrm{E}-02$ & 5.8501E-02 \\
\hline & & Rastrigin & $1.4218 \mathrm{E}+00$ & $2.1806 \mathrm{E}+00$ & $1.6717 \mathrm{E}+00$ & $1.6749 \mathrm{E}+00$ \\
\hline & & Rosenbrock & $8.2860 \mathrm{E}-01$ & $1.9936 \mathrm{E}+00$ & $1.5493 \mathrm{E}+00$ & $1.4291 \mathrm{E}+00$ \\
\hline & & Sphere & $1.0292 \mathrm{E}+00$ & $2.5103 \mathrm{E}+00$ & $1.6459 \mathrm{E}+00$ & $1.5085 \mathrm{E}+00$ \\
\hline & \multirow{5}{*}{15} & Ackley & $5.1951 \mathrm{E}+00$ & $5.2854 \mathrm{E}+00$ & $5.3153 \mathrm{E}+00$ & $1.6518 \mathrm{E}+01$ \\
\hline & & Alpine & $2.7135 \mathrm{E}-02$ & $3.0368 \mathrm{E}-02$ & $2.8221 \mathrm{E}-02$ & $1.2297 \mathrm{E}-01$ \\
\hline & & Rastrigin & $4.9696 \mathrm{E}+00$ & $5.3201 \mathrm{E}+00$ & $5.0698 \mathrm{E}+00$ & $1.6802 \mathrm{E}+01$ \\
\hline & & Rosenbrock & $3.4887 \mathrm{E}+00$ & $3.1670 \mathrm{E}+00$ & $4.1914 \mathrm{E}+00$ & $1.4729 \mathrm{E}+01$ \\
\hline & & Sphere & $4.3385 \mathrm{E}+00$ & $5.2085 \mathrm{E}+00$ & $5.0122 \mathrm{E}+00$ & $1.6925 \mathrm{E}+01$ \\
\hline & \multirow{5}{*}{25} & Ackley & 7.6393E+00 & $7.6793 \mathrm{E}+00$ & $7.6874 \mathrm{E}+00$ & $2.4671 \mathrm{E}+01$ \\
\hline & & Alpine & $7.8720 \mathrm{E}-02$ & $4.2787 \mathrm{E}-02$ & $4.6770 \mathrm{E}-02$ & $3.3699 \mathrm{E}+00$ \\
\hline & & Rastrigin & $7.6441 \mathrm{E}+00$ & $7.6861 \mathrm{E}+00$ & $7.6774 \mathrm{E}+00$ & $2.5255 \mathrm{E}+01$ \\
\hline & & Rosenbrock & $7.6414 \mathrm{E}+00$ & $7.6490 \mathrm{E}+00$ & $7.6710 \mathrm{E}+00$ & $2.4731 \mathrm{E}+01$ \\
\hline & & Sphere & $7.6353 \mathrm{E}+00$ & $7.6774 \mathrm{E}+00$ & $7.6694 \mathrm{E}+00$ & $2.4498 \mathrm{E}+01$ \\
\hline
\end{tabular}


Y.V.R. Naga Pawan et al., International Journal of Emerging Trends in Engineering Research, 8(9), September 2020, 6111 - 6130

Comparison of PSOs with Mean Time (in Secs)
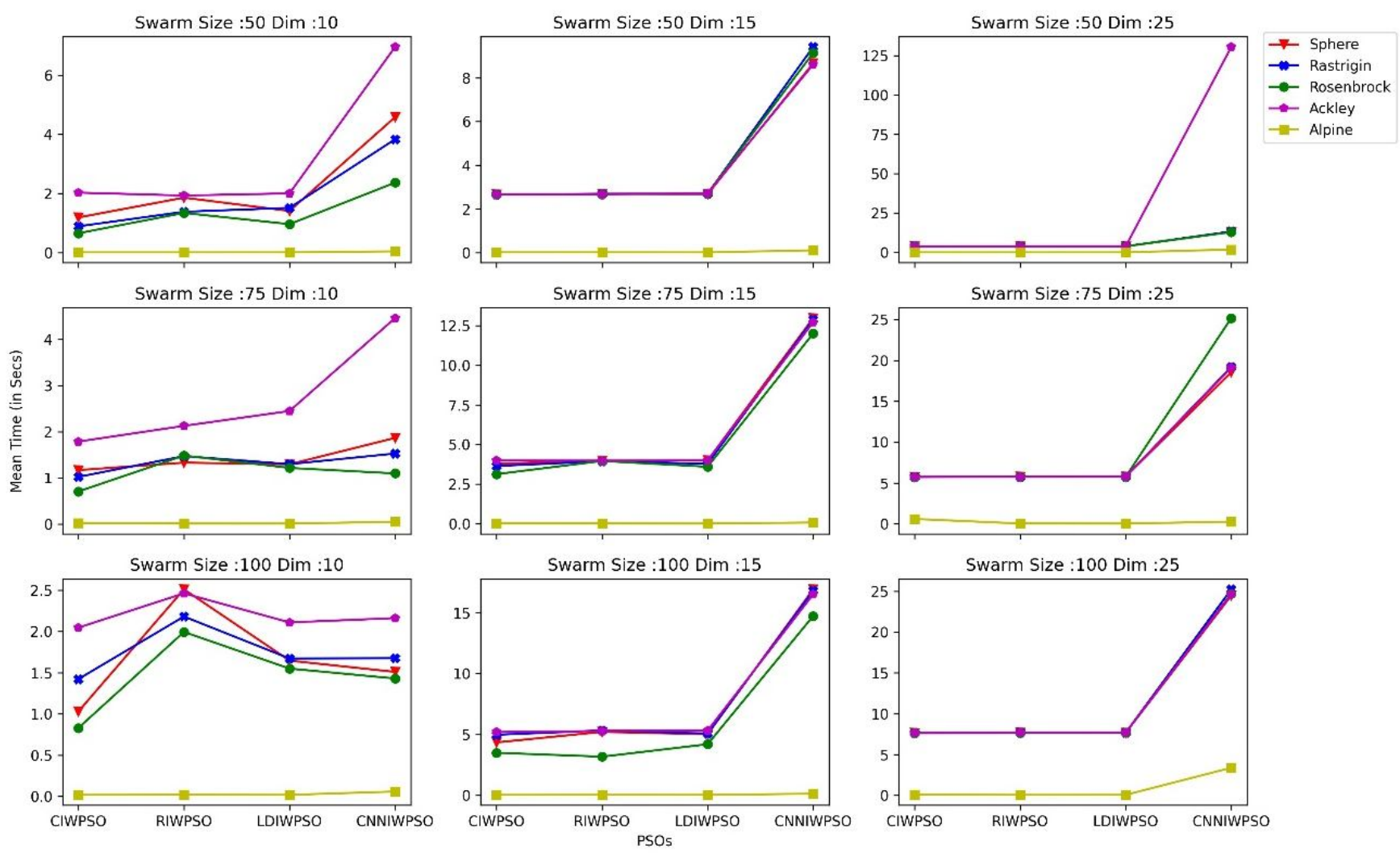

Figure 9: Mean Time computed for the swarm size of 50, 75 and 100 with dimensions 10, 15 and 25

From the Table 7 and Figure 9, the mean time for CNNIWPSO is non-paying when compared with other methods for the benchmarks considered. 
Y.V.R. Naga Pawan et al., International Journal of Emerging Trends in Engineering Research, 8(9), September 2020, 6111 - 6130

8: Computed MSE for PSOs with respect to different Swarm Sizes and Dimensions. (Figure 10)

\begin{tabular}{|c|c|c|c|c|c|c|}
\hline \multirow{2}{*}{ Swarm Size } & \multirow{2}{*}{ Dimension } & \multirow{2}{*}{ BMF } & \multicolumn{4}{|c|}{ PSOs } \\
\hline & & & CIWPSO & RIWPSO & LDIWPSO & CNNIWPSO \\
\hline \multirow{15}{*}{50} & \multirow{5}{*}{10} & Ackley & $2.0873 \mathrm{E}+03$ & $8.6493 \mathrm{E}+02$ & $5.2874 \mathrm{E}+03$ & $2.3442 \mathrm{E}+03$ \\
\hline & & Alpine & $6.1994 \mathrm{E}+03$ & $4.4939 \mathrm{E}+03$ & $6.6229 \mathrm{E}+03$ & $4.9175 \mathrm{E}+03$ \\
\hline & & Rastrigin & $2.5925 \mathrm{E}+00$ & $9.4619 \mathrm{E}-01$ & $3.8737 \mathrm{E}+00$ & $2.5128 \mathrm{E}+00$ \\
\hline & & Rosenbrock & $1.3862 \mathrm{E}-01$ & 3.8367E-02 & $1.7532 \mathrm{E}-01$ & $6.5912 \mathrm{E}-02$ \\
\hline & & Sphere & $2.8601 \mathrm{E}+00$ & 8.6317E-01 & $4.1424 \mathrm{E}+00$ & $1.9725 \mathrm{E}+00$ \\
\hline & \multirow{5}{*}{15} & Ackley & 7.9797E+03 & $4.2119 \mathrm{E}+03$ & $1.7364 \mathrm{E}+04$ & $1.1199 \mathrm{E}+04$ \\
\hline & & Alpine & $2.0962 \mathrm{E}+04$ & $1.2367 \mathrm{E}+04$ & $2.4993 \mathrm{E}+04$ & $1.6756 \mathrm{E}+04$ \\
\hline & & Rastrigin & $5.0303 \mathrm{E}+00$ & $2.5284 \mathrm{E}+00$ & $1.1421 \mathrm{E}+01$ & $9.1878 \mathrm{E}+00$ \\
\hline & & Rosenbrock & $1.7988 \mathrm{E}-01$ & $6.3481 \mathrm{E}-02$ & $2.8338 \mathrm{E}-01$ & $1.8763 \mathrm{E}-01$ \\
\hline & & Sphere & $7.4684 \mathrm{E}+00$ & $2.7028 \mathrm{E}+00$ & $1.0243 \mathrm{E}+01$ & $6.1282 \mathrm{E}+00$ \\
\hline & \multirow{5}{*}{25} & Ackley & $4.5230 \mathrm{E}+04$ & $2.1847 \mathrm{E}+04$ & $7.1614 \mathrm{E}+04$ & $5.0969 \mathrm{E}+04$ \\
\hline & & Alpine & $4.4880 \mathrm{E}+04$ & $1.2506 \mathrm{E}+04$ & $6.2091 \mathrm{E}+04$ & $6.1277 \mathrm{E}+04$ \\
\hline & & Rastrigin & $2.9302 \mathrm{E}+01$ & $1.4082 \mathrm{E}+01$ & $5.7999 \mathrm{E}+01$ & $3.6505 \mathrm{E}+01$ \\
\hline & & Rosenbrock & 7.9662E-01 & 3.6970E-01 & $1.3404 \mathrm{E}+00$ & $8.0071 \mathrm{E}-01$ \\
\hline & & Sphere & $3.2779 \mathrm{E}+01$ & $1.5244 \mathrm{E}+01$ & $5.4221 \mathrm{E}+01$ & $3.5839 \mathrm{E}+01$ \\
\hline \multirow{15}{*}{75} & \multirow{5}{*}{10} & Ackley & $3.2658 \mathrm{E}+03$ & $1.1920 \mathrm{E}+03$ & $3.7526 \mathrm{E}+03$ & $1.6681 \mathrm{E}+03$ \\
\hline & & Alpine & $4.2792 \mathrm{E}+03$ & $5.0463 \mathrm{E}+03$ & $6.3290 \mathrm{E}+03$ & $5.5003 \mathrm{E}+03$ \\
\hline & & Rastrigin & $3.5056 \mathrm{E}+00$ & $2.9337 \mathrm{E}+00$ & $4.3236 \mathrm{E}+00$ & $2.6990 \mathrm{E}+00$ \\
\hline & & Rosenbrock & $1.2335 \mathrm{E}-01$ & $1.1267 \mathrm{E}-01$ & $1.3074 \mathrm{E}-01$ & $6.0666 \mathrm{E}-02$ \\
\hline & & Sphere & $2.7966 \mathrm{E}+00$ & $1.9399 \mathrm{E}+00$ & $6.6485 \mathrm{E}+00$ & $2.2977 \mathrm{E}+00$ \\
\hline & \multirow{5}{*}{15} & Ackley & $6.4571 \mathrm{E}+03$ & $2.8732 \mathrm{E}+03$ & $1.1255 \mathrm{E}+04$ & $8.6502 \mathrm{E}+03$ \\
\hline & & Alpine & $1.8329 \mathrm{E}+04$ & $1.2123 \mathrm{E}+04$ & $2.2842 \mathrm{E}+04$ & $2.1777 \mathrm{E}+04$ \\
\hline & & Rastrigin & $6.0688 \mathrm{E}+00$ & $1.8643 \mathrm{E}+00$ & $8.0952 \mathrm{E}+00$ & $6.2620 \mathrm{E}+00$ \\
\hline & & Rosenbrock & $1.5713 \mathrm{E}-01$ & $5.2273 \mathrm{E}-02$ & $2.3691 \mathrm{E}-01$ & $1.2508 \mathrm{E}-01$ \\
\hline & & Sphere & $4.9168 \mathrm{E}+00$ & $2.0028 \mathrm{E}+00$ & $9.2308 \mathrm{E}+00$ & $5.5679 \mathrm{E}+00$ \\
\hline & \multirow{5}{*}{25} & Ackley & 3.9437E+04 & $1.5679 \mathrm{E}+04$ & $6.2383 \mathrm{E}+04$ & $4.6779 \mathrm{E}+04$ \\
\hline & & Alpine & $1.3129 \mathrm{E}+04$ & $3.3265 \mathrm{E}+04$ & $6.8909 \mathrm{E}+04$ & $5.2392 \mathrm{E}+04$ \\
\hline & & Rastrigin & $2.6611 \mathrm{E}+01$ & $9.2596 \mathrm{E}+00$ & $4.0828 \mathrm{E}+01$ & $2.9321 \mathrm{E}+01$ \\
\hline & & Rosenbrock & $5.4986 \mathrm{E}-01$ & $2.5714 \mathrm{E}-01$ & $1.0092 \mathrm{E}+00$ & $8.2196 \mathrm{E}-01$ \\
\hline & & Sphere & $2.5052 \mathrm{E}+01$ & $1.0693 \mathrm{E}+01$ & $3.9131 \mathrm{E}+01$ & $2.7746 \mathrm{E}+01$ \\
\hline \multirow{15}{*}{100} & \multirow{5}{*}{10} & Ackley & $2.4215 \mathrm{E}+03$ & $2.5293 \mathrm{E}+03$ & $4.5860 \mathrm{E}+03$ & $2.3795 \mathrm{E}+03$ \\
\hline & & Alpine & $7.5392 \mathrm{E}+03$ & $3.3331 \mathrm{E}+03$ & $6.8450 \mathrm{E}+03$ & $6.4054 \mathrm{E}+03$ \\
\hline & & Rastrigin & $1.9976 \mathrm{E}+00$ & $2.0451 \mathrm{E}+00$ & $4.0036 \mathrm{E}+00$ & $2.0872 \mathrm{E}+00$ \\
\hline & & Rosenbrock & $1.0102 \mathrm{E}-01$ & 7.5227E-02 & $1.0819 \mathrm{E}-01$ & $5.0906 \mathrm{E}-02$ \\
\hline & & Sphere & $2.7598 \mathrm{E}+00$ & $3.1286 \mathrm{E}+00$ & $4.9039 \mathrm{E}+00$ & $1.6739 \mathrm{E}+00$ \\
\hline & \multirow{5}{*}{15} & Ackley & $6.0384 \mathrm{E}+03$ & $2.1032 \mathrm{E}+03$ & $1.1749 \mathrm{E}+04$ & $6.4390 \mathrm{E}+03$ \\
\hline & & Alpine & $2.1144 \mathrm{E}+04$ & $1.1666 \mathrm{E}+04$ & $2.0460 \mathrm{E}+04$ & $1.9025 \mathrm{E}+04$ \\
\hline & & Rastrigin & $4.2744 \mathrm{E}+00$ & $1.4981 \mathrm{E}+00$ & $7.0306 \mathrm{E}+00$ & $4.1351 \mathrm{E}+00$ \\
\hline & & Rosenbrock & $1.3655 \mathrm{E}-01$ & 3.9989E-02 & $2.5110 \mathrm{E}-01$ & $1.6966 \mathrm{E}-01$ \\
\hline & & Sphere & $5.1906 \mathrm{E}+00$ & $1.7117 \mathrm{E}+00$ & $8.3602 \mathrm{E}+00$ & $5.5858 \mathrm{E}+00$ \\
\hline & \multirow{5}{*}{25} & Ackley & $3.2916 \mathrm{E}+04$ & $1.2923 \mathrm{E}+04$ & $4.7496 \mathrm{E}+04$ & $3.3739 \mathrm{E}+04$ \\
\hline & & Alpine & $4.6683 \mathrm{E}+04$ & $1.1999 \mathrm{E}+04$ & $6.6734 \mathrm{E}+04$ & $7.2207 \mathrm{E}+04$ \\
\hline & & Rastrigin & $1.9194 \mathrm{E}+01$ & $9.2106 \mathrm{E}+00$ & $3.5866 \mathrm{E}+01$ & $2.1958 \mathrm{E}+01$ \\
\hline & & Rosenbrock & $5.2088 \mathrm{E}-01$ & $2.2935 \mathrm{E}-01$ & $7.7648 \mathrm{E}-01$ & $7.1243 \mathrm{E}-01$ \\
\hline & & Sphere & $1.8964 \mathrm{E}+01$ & $9.0607 \mathrm{E}+00$ & $3.1134 \mathrm{E}+01$ & $1.7543 \mathrm{E}+01$ \\
\hline
\end{tabular}


Y.V.R. Naga Pawan et al., International Journal of Emerging Trends in Engineering Research, 8(9), September 2020, 6111 - 6130

Comparison of PSOs with MSE
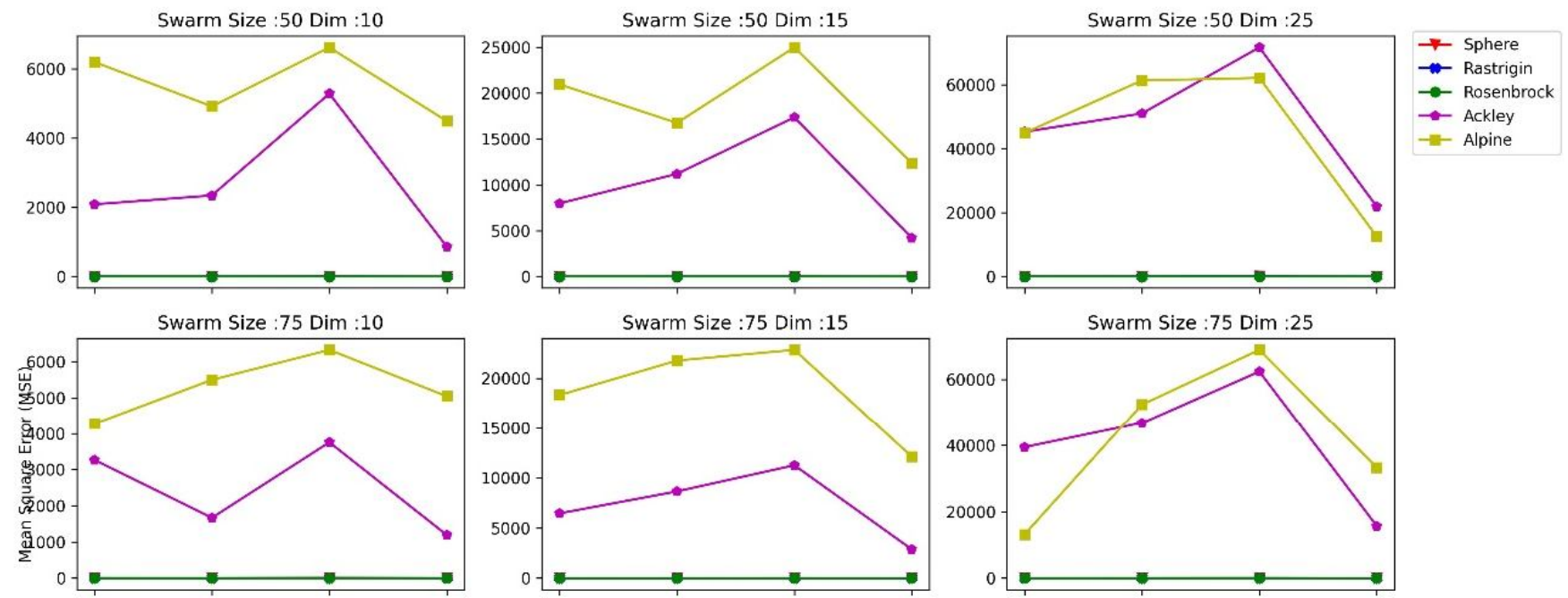

Swarm Size : 75 Dim :25

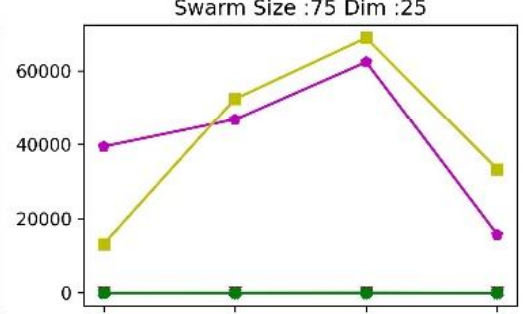

Swarm Size :100 Dim :10

Swarm Size :100 Dim :15

Swarm Size :100 Dim :25
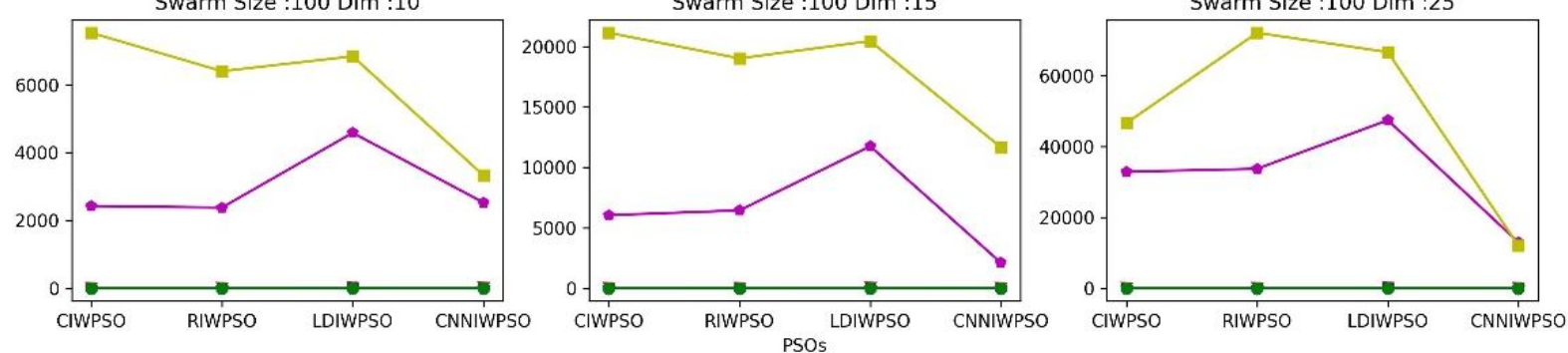

Figure 10: Mean Square Error (MSE) computed for the swarm size of 50, 75 and 100 with dimensions 10, 15 and 25 
Y.V.R. Naga Pawan et al., International Journal of Emerging Trends in Engineering Research, 8(9), September 2020, 6111 - 6130

Table 9: Computed RMSE for PSOs with respect different Swarm Sizes and Dimensions. (Figure 11)

\begin{tabular}{|c|c|c|c|c|c|c|}
\hline \multirow{2}{*}{$\begin{array}{c}\text { Swarm } \\
\text { Size }\end{array}$} & \multirow{2}{*}{ Dimension } & \multirow{2}{*}{ BMF } & \multicolumn{4}{|c|}{ PSOs } \\
\hline & & & CIWPSO & RIWPSO & LDIWPSO & CNNIWPSO \\
\hline \multirow{15}{*}{50} & \multirow{5}{*}{10} & Ackley & $4.5687 \mathrm{E}+01$ & $2.9410 \mathrm{E}+01$ & $7.2714 \mathrm{E}+01$ & $4.8417 \mathrm{E}+01$ \\
\hline & & Alpine & $7.8736 \mathrm{E}+01$ & $6.7036 \mathrm{E}+01$ & $8.1381 \mathrm{E}+01$ & $7.0125 \mathrm{E}+01$ \\
\hline & & Rastrigin & $1.6101 \mathrm{E}+00$ & $9.7272 \mathrm{E}-01$ & $1.9682 \mathrm{E}+00$ & $1.5852 \mathrm{E}+00$ \\
\hline & & Rosenbrock & $3.7232 \mathrm{E}-01$ & $1.9587 \mathrm{E}-01$ & $4.1871 \mathrm{E}-01$ & $2.5673 \mathrm{E}-01$ \\
\hline & & Sphere & $1.6912 \mathrm{E}+00$ & $9.2907 \mathrm{E}-01$ & $2.0353 \mathrm{E}+00$ & $1.4045 \mathrm{E}+00$ \\
\hline & \multirow{5}{*}{15} & Ackley & $8.9329 \mathrm{E}+01$ & $6.4899 \mathrm{E}+01$ & $1.3177 \mathrm{E}+02$ & $1.0582 \mathrm{E}+02$ \\
\hline & & Alpine & $1.4478 \mathrm{E}+02$ & $1.1121 \mathrm{E}+02$ & $1.5809 \mathrm{E}+02$ & $1.2944 \mathrm{E}+02$ \\
\hline & & Rastrigin & $2.2428 \mathrm{E}+00$ & $1.5901 \mathrm{E}+00$ & $3.3796 \mathrm{E}+00$ & $3.0311 \mathrm{E}+00$ \\
\hline & & Rosenbrock & $4.2412 \mathrm{E}-01$ & $2.5196 \mathrm{E}-01$ & $5.3234 \mathrm{E}-01$ & $4.3316 \mathrm{E}-01$ \\
\hline & & Sphere & $2.7328 \mathrm{E}+00$ & $1.6440 \mathrm{E}+00$ & $3.2005 E+00$ & $2.4755 \mathrm{E}+00$ \\
\hline & \multirow{5}{*}{25} & Ackley & $2.1267 \mathrm{E}+02$ & $1.4781 \mathrm{E}+02$ & $2.6761 \mathrm{E}+02$ & $2.2576 \mathrm{E}+02$ \\
\hline & & Alpine & $2.1185 \mathrm{E}+02$ & $1.1183 \mathrm{E}+02$ & $2.4918 \mathrm{E}+02$ & $2.4754 \mathrm{E}+02$ \\
\hline & & Rastrigin & $5.4131 \mathrm{E}+00$ & $3.7526 \mathrm{E}+00$ & $7.6157 \mathrm{E}+00$ & $6.0420 \mathrm{E}+00$ \\
\hline & & Rosenbrock & $8.9254 \mathrm{E}-01$ & $6.0803 \mathrm{E}-01$ & $1.1578 \mathrm{E}+00$ & 8.9483E-01 \\
\hline & & Sphere & $5.7253 \mathrm{E}+00$ & $3.9044 \mathrm{E}+00$ & $7.3635 \mathrm{E}+00$ & $5.9866 \mathrm{E}+00$ \\
\hline \multirow{15}{*}{75} & \multirow{5}{*}{10} & Ackley & $5.7147 \mathrm{E}+01$ & $3.4526 \mathrm{E}+01$ & $6.1259 \mathrm{E}+01$ & $4.0842 \mathrm{E}+01$ \\
\hline & & Alpine & $6.5416 \mathrm{E}+01$ & $7.1037 \mathrm{E}+01$ & $7.9555 \mathrm{E}+01$ & $7.4164 \mathrm{E}+01$ \\
\hline & & Rastrigin & $1.8723 \mathrm{E}+00$ & $1.7128 \mathrm{E}+00$ & $2.0793 \mathrm{E}+00$ & $1.6429 \mathrm{E}+00$ \\
\hline & & Rosenbrock & $3.5122 \mathrm{E}-01$ & 3.3567E-01 & $3.6158 \mathrm{E}-01$ & $2.4631 \mathrm{E}-01$ \\
\hline & & Sphere & $1.6723 \mathrm{E}+00$ & $1.3928 \mathrm{E}+00$ & $2.5785 \mathrm{E}+00$ & $1.5158 \mathrm{E}+00$ \\
\hline & \multirow{5}{*}{15} & Ackley & $8.0356 \mathrm{E}+01$ & $5.3602 \mathrm{E}+01$ & $1.0609 \mathrm{E}+02$ & $9.3006 \mathrm{E}+01$ \\
\hline & & Alpine & $1.3538 \mathrm{E}+02$ & $1.1010 \mathrm{E}+02$ & $1.5114 \mathrm{E}+02$ & $1.4757 \mathrm{E}+02$ \\
\hline & & Rastrigin & $2.4635 \mathrm{E}+00$ & $1.3654 \mathrm{E}+00$ & $2.8452 \mathrm{E}+00$ & $2.5024 \mathrm{E}+00$ \\
\hline & & Rosenbrock & $3.9639 \mathrm{E}-01$ & $2.2863 \mathrm{E}-01$ & $4.8673 \mathrm{E}-01$ & 3.5367E-01 \\
\hline & & Sphere & $2.2174 \mathrm{E}+00$ & $1.4152 \mathrm{E}+00$ & $3.0382 \mathrm{E}+00$ & $2.3596 \mathrm{E}+00$ \\
\hline & \multirow{5}{*}{25} & Ackley & $1.9859 \mathrm{E}+02$ & $1.2522 \mathrm{E}+02$ & $2.4977 \mathrm{E}+02$ & $2.1628 \mathrm{E}+02$ \\
\hline & & Alpine & $1.1458 \mathrm{E}+02$ & $1.8239 \mathrm{E}+02$ & $2.6251 \mathrm{E}+02$ & $2.2889 \mathrm{E}+02$ \\
\hline & & Rastrigin & $5.1586 \mathrm{E}+00$ & $3.0430 \mathrm{E}+00$ & $6.3897 \mathrm{E}+00$ & $5.4149 \mathrm{E}+00$ \\
\hline & & Rosenbrock & 7.4153E-01 & $5.0709 \mathrm{E}-01$ & $1.0046 \mathrm{E}+00$ & $9.0662 \mathrm{E}-01$ \\
\hline & & Sphere & $5.0052 \mathrm{E}+00$ & $3.2700 \mathrm{E}+00$ & $6.2555 \mathrm{E}+00$ & $5.2674 \mathrm{E}+00$ \\
\hline \multirow{15}{*}{100} & \multirow{5}{*}{10} & Ackley & $4.9209 \mathrm{E}+01$ & $5.0292 \mathrm{E}+01$ & $6.7720 \mathrm{E}+01$ & $4.8780 \mathrm{E}+01$ \\
\hline & & Alpine & $8.6829 \mathrm{E}+01$ & $5.7733 \mathrm{E}+01$ & $8.2734 \mathrm{E}+01$ & $8.0034 \mathrm{E}+01$ \\
\hline & & Rastrigin & $1.4134 \mathrm{E}+00$ & $1.4301 \mathrm{E}+00$ & $2.0009 \mathrm{E}+00$ & $1.4447 \mathrm{E}+00$ \\
\hline & & Rosenbrock & $3.1783 \mathrm{E}-01$ & $2.7428 \mathrm{E}-01$ & $3.2893 \mathrm{E}-01$ & $2.2562 \mathrm{E}-01$ \\
\hline & & Sphere & $1.6613 \mathrm{E}+00$ & $1.7688 \mathrm{E}+00$ & $2.2145 \mathrm{E}+00$ & $1.2938 \mathrm{E}+00$ \\
\hline & \multirow{5}{*}{15} & Ackley & $7.7707 \mathrm{E}+01$ & $4.5860 \mathrm{E}+01$ & $1.0839 \mathrm{E}+02$ & $8.0244 \mathrm{E}+01$ \\
\hline & & Alpine & $1.4541 \mathrm{E}+02$ & $1.0801 \mathrm{E}+02$ & $1.4304 \mathrm{E}+02$ & $1.3793 \mathrm{E}+02$ \\
\hline & & Rastrigin & $2.0675 \mathrm{E}+00$ & $1.2240 \mathrm{E}+00$ & $2.6515 \mathrm{E}+00$ & $2.0335 \mathrm{E}+00$ \\
\hline & & Rosenbrock & 3.6953E-01 & $1.9997 \mathrm{E}-01$ & $5.0110 \mathrm{E}-01$ & $4.1190 \mathrm{E}-01$ \\
\hline & & Sphere & $2.2783 \mathrm{E}+00$ & $1.3083 \mathrm{E}+00$ & $2.8914 \mathrm{E}+00$ & $2.3634 \mathrm{E}+00$ \\
\hline & \multirow{5}{*}{25} & Ackley & $1.8143 \mathrm{E}+02$ & $1.1368 \mathrm{E}+02$ & $2.1794 \mathrm{E}+02$ & $1.8368 \mathrm{E}+02$ \\
\hline & & Alpine & $2.1606 \mathrm{E}+02$ & $1.0954 \mathrm{E}+02$ & $2.5833 \mathrm{E}+02$ & $2.6871 \mathrm{E}+02$ \\
\hline & & Rastrigin & $4.3811 \mathrm{E}+00$ & $3.0349 \mathrm{E}+00$ & $5.9888 \mathrm{E}+00$ & $4.6859 \mathrm{E}+00$ \\
\hline & & Rosenbrock & $7.2172 \mathrm{E}-01$ & $4.7890 \mathrm{E}-01$ & $8.8118 \mathrm{E}-01$ & $8.4406 \mathrm{E}-01$ \\
\hline & & Sphere & $4.3548 \mathrm{E}+00$ & $3.0101 \mathrm{E}+00$ & $5.5798 \mathrm{E}+00$ & $4.1884 \mathrm{E}+00$ \\
\hline
\end{tabular}


Comparison of PSOs with RMSE
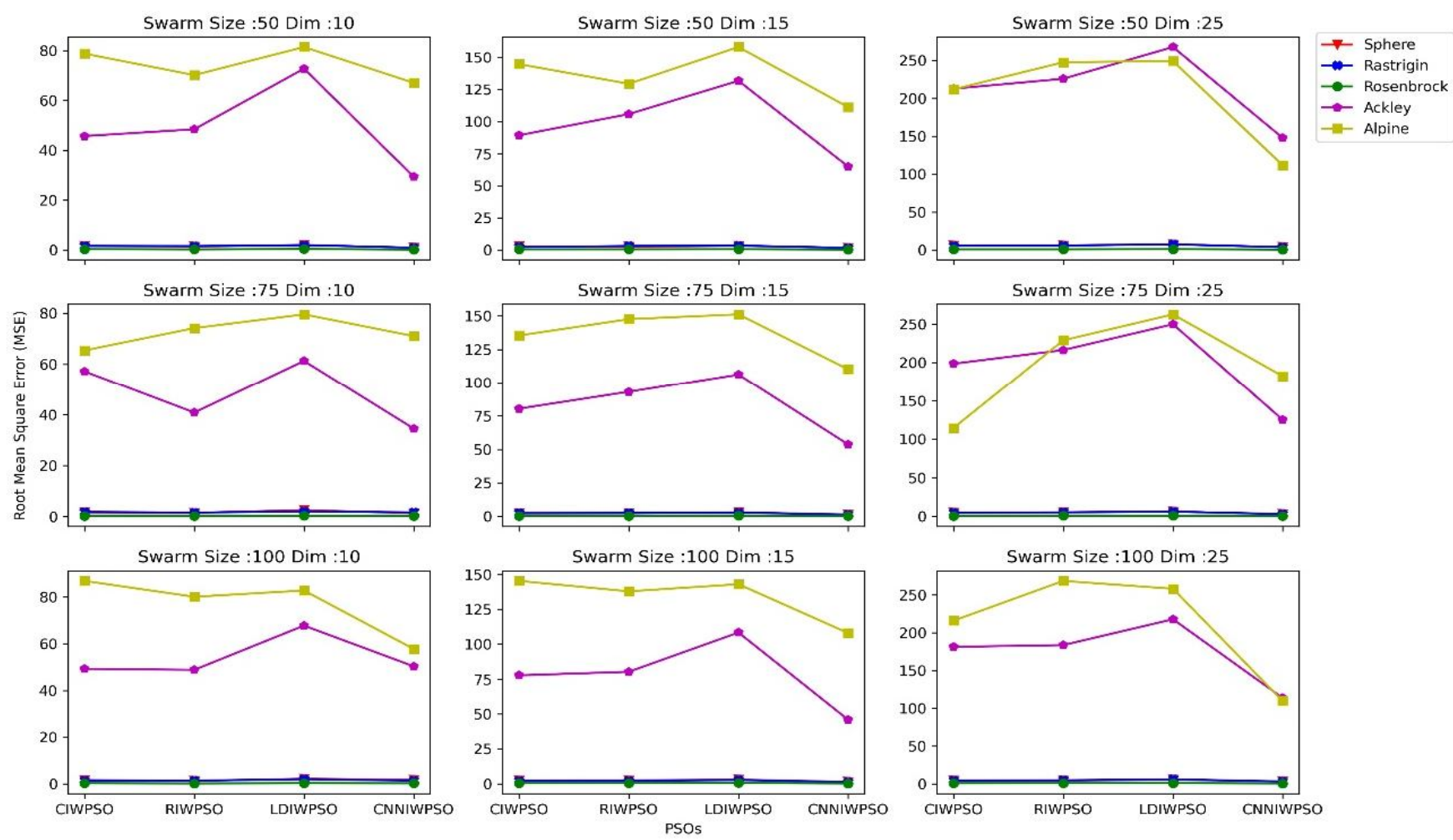

Figure 11: Root Mean Square Error (RMSE) computed for the swarm size of 50, 75 and 100 with dimensions 10, 15 and 25

The MSE and RMSE values are finer for CNNIWPSO, from the Table 8, Table 9, Figure 10 and Figure 11 for the benchmark functions.

CNNIWPSO is delivered better results over CIWPSO, RIWPSO and LDIWPSO from the perspective of best error, mean error, variance \& standard deviation, mean iterations and, MSE \& RMSE.

\section{CONCLUSION AND FUTURE WORK}

This paper presents a new inertia weight based PSO using Convolutional Neural Networks (CNN). A set of 5 most common optimization test problems and eight criteria are considered. The overall outcome shows that CNNIWPSO is progressive with CIWPSO, RIWPSO and LDIWPSO.

In future, the parameters of CNN are tuned to improve the performance and also experiments with larger swarm sizes and dimension are conducted to understand CNNIWPSO performance. There is scope for using CNNIWPSO in the optimization of various applications presented in [22] -[31].

\section{REFERENCES}

1. Kennedy J, Eberhart R.C., Particle Swarm Optimization. In: Proceedings of the IEEE International Conference on Neural networks, Perth Australia,1995,1942-1948
2. Y.Shi and R. Eberhart., A modified particle swarm optimizer, In Evolutionary Computation Proceedings, 1998. IEEE World Congress on Computational Intelligence., The 1998 IEEE International Conference on, pages 69-73. IEEE, 2002.

3. C. M. Huang, C. J. Huang and M. L. Wang, A Particle Swarm Optimization to Identifying the ARMAX Model for Short-Term Load Forecasting. IEEE Tran. on Power Systems, 20(2): 1126-1133, May 2005.

4. X. H. Hu, R. C. Eberhart, Y. H. Shi, Engineering optimization. with particle swarm. Proc. of the IEEE Swarm Intelligence Symposium, pages 53-57, 2003.

5. X. H. Hu, Y. H. Shi, R. Eberhart, Recent advances in particle swarm, Congress on Evolutionary Computation, pages 90-97, 2004.

6. M. Clerc, Particle Swarm Optimization, ISTE Publishing Company, 2006.

7. N. Nedjah, L. D. M. Mourelle, Systems Engineering Using Particle Swarm Optimization, Nova Science Publishers, 2007.

8. Yudong Zhang, Shuihua Wang, and Genlin Ji, A Comprehensive Particle Swarm Optimization Algorithm and Its Applications, Mathematical Problems in Engineering, Volume-2015, pp 1-38.

9. J. C. Bansal, P. K. Singh, M. Saraswat, A. Verma, S. S. Jadon and A. Abraham, Inertia Weight strategies in 
Y.V.R. Naga Pawan et al., International Journal of Emerging Trends in Engineering Research, 8(9), September 2020, 6111 - 6130

Particle Swarm Optimization, 2011 Third World Congress on Nature and Biologically Inspired Computing, Salamanca, 2011, pp. 633-640, doi: 10.1109/NaBIC.2011.6089659.

10. Ashok Kumar, Brajesh Kumar Singh, B.D.K.Patro, Particle Swarm Optimization: A Study of Variants and their applications, International Journal of Computer Applications, Volume 135 (5), November 2016, PP 24-30.

11. Riccardo Poli, "Analysis of the Publications on the Applications of Particle Swarm Optimisation", Journal of Artificial Evolution and Applications, Vol.2008, January, 2018, 10 Pages.

12. E. T. Oldewage, A. P. Engelbrecht and C. W. Cleghorn, The merits of velocity clamping particle swarm optimisation in high dimensional spaces, 2017 IEEE Symposium Series on Computational Intelligence (SSCI), Honolulu, HI, 2017, pp. 1-8, doi: 10.1109/SSCI.2017.8280887.

13. Frans van den Bergh. An Analysis of Particle Swarm Optimizers. PhD thesis, University of Pretoria, 2001.

14. Federico Marini, Beata Walczak, Particle swarm optimization (PSO). A tutorial, Chemometrics and Intelligent Laboratory Systems, Volume 149, Part B, 2015, Pages 153-165, ISSN 0169-7439, https://doi.org/10.1016/j.chemolab.2015.08.020.

15. J. C. Bansal, P. K. Singh, M. Saraswat, A. Verma, S. S. Jadon and A. Abraham, Inertia Weight strategies in Particle Swarm Optimization, 2011 Third World Congress on Nature and Biologically Inspired Computing, Salamanca, 2011, pp. 633-640, doi: 10.1109/NaBIC.2011.6089659.

16. Y. Shi and R. Eberhart., A modified particle swarm optimizer, In Evolutionary Computation Proceedings, 1998. IEEE World Congress on Computational Intelligence., The 1998 IEEE International Conference on, pages 69-73. IEEE, 2002.

17. R.C. Eberhart and Y. Shi., Tracking and optimizing dynamic systems with particle swarms, In Evolutionary Computation, 2001. Proceedings of the 2001 Congress on, volume 1, pages 94-100. IEEE, 2002.

18. J. Xin, G. Chen, and Y. Hai., A Particle Swarm Optimizer with Multistage Linearly-Decreasing Inertia Weight, In Computational Sciences and Optimization, 2009. CSO 2009. International Joint Conference on, volume 1, pages 505-508. IEEE, 2009.

19. [Kun Miao, Xiaolin Mao, Chen $\mathrm{Li}$, Individualism of particles in particle swarm optimization, Applied Soft Computing, Volume 83, 2019, 105619, ISSN 1568-4946, https://doi.org/10.1016/j.asoc.2019.105619.

20. Mewael Isiet, Mohamed Gadala, Self-adapting control parameters in particle swarm optimization, Applied Soft Computing, Volume 83, 2019, 105653, ISSN 1568-4946, https://doi.org/10.1016/j.asoc.2019.105653.

21. M. He, M. Liu, R. Wang et al., Particle swarm optimization with damping factor and cooperative mechanism, Applied Soft Computing Journal (2018), https://doi.org/10.1016/j.asoc.2018.11.050

22. Dharmapuri, S. L., Dandamudi, P. K., Botcha, V. M., \& Kolla, B. P. (2020). Detecting central nervous system disorder using machine learning technique (XGB classifier). International Journal of Emerging Trends in Engineering Research, 8(4), 1142-1147. doi:10.30534/ijeter/2020/31842020

23. Meghana, A. S., Sudhakar, S., Arumugam, G., Srinivasan, P., \& Prakash, K. B. (2020). Age and gender prediction using convolution, resnet50 and inception resnetv2. International Journal of Advanced Trends in Computer Science and Engineering, 9(2), 1328-1334. doi:10.30534/ijatcse/2020/65922020

24. Lakshmi, M., Sahithi, G. S., Pravallika, J. L., \& Prakash, K. B. (2019). Hand gesture identification and recognition using modern deep learning algorithms, International Journal of Engineering and Advanced Technology, 9(1), 5027-5031. doi:10.35940/ ijeat.A3004.109119

25. Krishna Sai, M., Lakshma Reddy, P., Krishna, N. S. R., Teja, P. V. N. S. R., \& Prakash, K. B. (2019), Hybrid encryption for medical applications using advanced algorithms in internet of things. Indian Journal of Public Health Research and Development, 10(6), 179-184. doi:10.5958/0976-5506.2019.01262.2

26. Kolla, B. P., \& Raman, A. R. (2019). Data engineered content extraction studies for indian web pages doi:10.1007/978-981-10-8055-5_45

27. Ismail, M., Prakash, K. B., \& Rao, M. N. (2018). Collaborative filtering-based recommendation of online social voting. International Journal of Engineering and Technology(UAE), 7(3), 1504-1507. doi:10.14419/ijet.v7i3.11630

28. Botcha, V. M., Monitha, G., Madala, D. N. S., \& Kolla, B. P. (2020). Analysis of nature inspired algorithms, Journal of Critical Reviews, 7(4), 752-754. doi: $10.31838 /$ jcr.07.04.140

29. Bharadwaj, Y. S. S., Rajaram, P., Sriram, V. P., Sudhakar, S., \& Prakash, K. B. (2020). Effective handwritten digit recognition using deep convolution neural network, International Journal of Advanced Trends in Computer Science and Engineering, 9(2), 1335-1339. doi:10.30534/ijatcse/2020/66922020

30. Prakash, K. B., Imambi, S. S., Ismail, M., Pavan Kumar, T., \& Naga Pawan, Y. V. R. (2020). Analysis, prediction and evaluation of covid-19 datasets using machine learning algorithms. International Journal of Emerging Trends in Engineering Research, 8(5), 2199-2204. doi:10.30534/ijeter/2020/117852020

31. Prakash, K. B., Nagapawan, Y. V. R., Kalyani, N. L., \& Kumar, V. P. (2020). Chatterbot implementation using transfer learning and LSTM encoder-decoder architecture, International Journal of Emerging Trends in Engineering Research, 8(5), 1709-1715. doi:10.30534/ijeter/2020/35852020 